\title{
Leguminosae-papilionoideae from the parque estadual das Várzeas do Rio Ivinhema, Mato Grosso do Sul State, Brazil*
}

\author{
Rafael Brune Caboco ${ }^{1,5}$, Thiago Prates Rolim ${ }^{2}$, Ângela Lúcia Bagnatori Sartori ${ }^{3}$ \& Alan Sciamarelli ${ }^{4}$ \\ ${ }^{1}$ Programa de Pós-graduação em Biologia Vegetal, Departamento de Biologia - DBI, \\ Centro de Ciências Biológicas e da Saúde - CCBS, Universidade Federal de Mato Grosso do Sul - UFMS, \\ Cidade Universitária, CP 549, CEP 79070-900, Campo Grande, MS, Brasil. http://dbi.ufms.br \\ ${ }^{2}$ Faculdade de Ciências Biológicas e da Saúde - FCBA, Universidade Federal da Grande Dourados - \\ UFGD, Rod. Dourados-Itahum, Km 12, CEP 79804-970, Dourados, MS, Brasil. http://www.ufgd.edu/fcba \\ ${ }^{3}$ Departamento de Biologia - DBI, Centro de Ciências Biológicas e da Saúde-CCBS, \\ Universidade Federal de Mato Grosso do Sul-UFMS, Cidade Universitária, CEP 79070-900, \\ CP 549, Campo Grande, MS, Brasil. http://dbi.ufms.br \\ ${ }^{4}$ Departamento de Biologia, Universidade Federal da Grande Dourados - UFGD, Rod Dourados-Itahum, \\ Km 12, CEP 79804-970, Dourados, MS, Brasil. http://www.ufgd.edu/fcba \\ ${ }^{5}$ Corresponding author: Rafael Brune, e-mail: kakara@ibest.com.br \\ * Part of a Master's dissertation from the first author
}

CABOCO, R.B., ROLIM, T.P., SARTORI, Â.L.B. \& SCIAMARELLI A. Leguminosae-papilionoideae from the parque estadual das Várzeas do Rio Ivinhema, Mato Grosso do Sul State, Brazil. Biota Neotrop. 12(2): http://www.biotaneotropica.org.br/v12n1/en/abstract?article+bn01212022012

\begin{abstract}
This study aims to evaluate the richness of the Papilionoideae species found in the Parque Estadual das Várzeas do Rio Ivinhema (PEVRI), which is located in the southeast region of Mato Grosso do Sul, Brazil, and investigate if the habits of this Leguminosae group vary in different formations of the park. Monthly collections, including all habits, were made by walking along pre-established trails, from September 2004 to September 2009. The PEVRI includes Seasonal Semideciduous Forest, Seasonal Alluvial Semideciduous Forest and fields. Twenty-three species distributed in 16 genera and 5 tribes were recorded. Phaseoleae was noted with 7 genera, followed by Dalbergieae with 6 genera and Desmodieae, Indigofereae and Crotalarieae with only 1 genus in each species. Among the 16 recorded genera, Crotalaria L. (4), Aeschynomene L. (3), Desmodium Desv., and Vigna Savi (2) were the most representative. The Papilionoideae with herbaceous and subshrub habits were predominant in humid and pastures, while arboreous species and lianas were recorded in the Seasonal Semideciduous Forest. Nine species of Papilionoideae were recorded in humid grasslands, eight species in Seasonal Semideciduous Forest, and only one in Alluvial Semideciduous Forest. Papilionoideae found in the PEVRI have also been recorded in cerrado, Seasonal Semideciduous Forest and Pantanal, except Andira inermis, which does not present a reference for Seasonal Alluvial Semideciduous Forest.

Keywords: fabaceae, leguminosae, floristic, Central-Western region, Brazil.
\end{abstract}

CABOCO, R.B., ROLIM, T.P., SARTORI, Â.L.B. \& SCIAMARELLI A. Papilionoideae do Parque Estadual das Várzeas do Rio Ivinhema, Mato Grosso do Sul, Brasil. Biota Neotrop. 12(2): http://www.biotaneotropica. org.br/v12n2/pt/abstract?article+bn01212022012

Resumo: Este estudo consiste no inventário das Leguminosae-Papilionoideae do Parque Estadual das Várzeas do Rio Ivinhema (PEVRI), localizado ao sudeste de Mato Grosso do Sul e na investigação do hábito predominante das espécies nas diferentes formações do parque. As coletas incluindo todos os hábitos foram realizadas por meio de caminhadas, entre setembro de 2004 a setembro de 2009. O PEVRI agrega remanescentes de Floresta Estacional Semidecídua, de Floresta Estacional Semidecídua Aluvial e de campos. Foram registradas 23 espécies reunidas em 16 gêneros e 5 tribos com destaque para Phaseoleae com 7 gêneros, seguida por Dalbergieae com 6 gêneros e Desmodieae, Indigofereae e Crotalarieae representadas por 1 gênero cada. Dentre os 16 gêneros registrados destacaram-se como mais representativos: Crotalaria L. (4), Aeschynomene L. (3), Desmodium Desv. e Vigna Savi (2). As Papilionoideae herbáceas e subarbustivas predominaram em campo úmido e campo com pastagens, as arbóreas e lianas em Floresta Estacional Semidecídua. Nove espécies de Papilionoideae foram registradas em campo úmido, oito em Floresta Estacional Semidecídua e apenas uma em Floresta Estacional Semidecídua Aluvial. As Papilionoideae do PEVRI também foram registradas em outros estudos no Cerrado, Floresta Estacional Semidecídua e Pantanal, exceto Andira inermis, sem registros para a Floresta Semidecídua Aluvial.

Palavras-chave: fabaceae, leguminosae, florística, região Centro Oeste, Brasil. 


\section{Introduction}

Leguminosae Adans., the third largest eudicotyledon family in the world (Lewis et al. 2005) and the first in Brazil (Forzza et al. 2010), mainly occurs in tropical and subtropical regions with approximately 727 genera and 19.325 species (Lewis et al. 2005). About 2.694 native species occur in Brazil, and they are distributed among 210 genera (Lima et al. 2010). This family is important to the economy, as well as to ecological studies, and many their representatives are capable of developing nodules with $\mathrm{N}_{2}$-fixing bacteria, while others have the potential to be used in agro forestry systems, thus having implications for the recovery of degraded areas and the maintenance of soil sustainability (Herrera et al. 1993, Franco \& Faria 1997). In taxonomic terms, the subfamilies Papilionoideae and Mimosoideae are supported as monophyletic but they are sheltered in the Caesalpinioideae a subfamily which is paraphiletic (Queiroz 2009).

The Papilionoideae comprises about 13.800 species widely distributed in tropical forests in arid and cold regions (Lewis et al. 2005). In Mato Grosso do Sul, the members of this subfamily are considered important components from many plant communities, occurring in several regions and in such different formations as Planalto Residual do Urucum (Damasceno-Junior unpublished data), semideciduous forest (Sciamarelli, unpublished data), Cerrado (Pott $\&$ Pott 1994) and Chaco (Noguchi et al. 2009). Some inventories in Mato Grosso do Sul are specifically referred to Aeschynomene (Lima et al. 2006), Desmodium (Nobre et al. 2008), Machaerium (Polido \& Sartori 2007, 2011) and Stylosanthes (Costas et al. 2008), but, so far, no study has specifically focused on all members of Papilionoideae occurring in a same area.

The present study aims (i) to evaluate the species richness of Papilionoideae in Parque Estadual das Várzeas do Rio Ivinhema (PEVRI), (ii) investigate the variation of the habits of this group in different formations of the park, and (iii) supply the key identification, descriptions, illustrations, taxonomic comments and information about the geographical distribution.

\section{Material and Methods}

\section{Study area}

The PEVRI is located in Mato Grosso do Sul, between Iguatemi and Nova Andradina micro-regions, and comprises Naviraí, Jatei and Taquarussu municipalities (Figure 1). It covers an area of 73,315 ha, $\left(22^{\circ} 55^{\prime} 14.8^{\prime \prime} \mathrm{S}\right.$ and $\left.53^{\circ} 39^{\prime} 13.1^{\prime \prime} \mathrm{W}\right)$ at $300 \mathrm{~m}$ mean altitude (Mato Grosso do Sul 2001). The climate is Cwa with dry winters and rainy summers (Köppen 1948). The average annual temperature is $22^{\circ} \mathrm{C}$, and the rainfall varies from 1400 to $1700 \mathrm{~mm}$. November, December and January are the wettest months (Oliveira \& Luzardo 2000).

The Parque Estadual das Várzeas do Rio Ivinhema (PEVRI) is the only one located in the Paraná basin, which is located in the southeast region of the state. The PEVRI woods belong to the Atlantic Forest, and, according to Veloso et al. (1991), it may be divided into Seasonal Alluvial Semideciduous Forest, which are mixed with humid grasslands, riparian forests and secondary formation. There is also some areas represented by Cerrado (Campos \& Souza 1997).

\section{Collection and analysis of the botanical material}

The collections were carried out from monthly April 2004 to September 2006, bimonthly July 2008 to September 2009, through non-systematic walks. During the collections, the general aspect of the plant, habitat, morphology and geographical coordinates were obtained.
The collected materials were deposited in the Herbarium CGMS of the Universidade Federal de Mato Grosso do Sul, and the duplicate were donated to the Herbarium of Dourados at UFGD/UEMS/ Unigran/Anhanguera and to the Herbarium UEC at the Universidade Estadual de Campinas. The identification of specimens was achieved using taxonomic literature, experts consultation, comparisons with other herbaria material and photographic analysis of the types. When necessary morphological information was achieved using specific literature sources.

To describe the types of habit, the terminology from GuedesBruni et al. (2002) was adopted with some modifications, as follows: herbs, non-woody plants up to $50 \mathrm{~cm}$ height; subshrubs, i.e., erect plants with ramifications near the soil and whose branches are partially lignified; shrubs, i.e., ramified plants $1 \mathrm{~m}$ in height and with woody branches; vines, defined as plants with pliable, non-woody branches that use trees or bushes for support; and lianas, i.e., various longstemmed, woody vines, and trees which were considered as plants due to their branches are near the apex, forming a woody shaft. The terminology used to describe the vegetative and reproductive structures followed Radford et al. (1974), Harris \& Harris (1994) and Barroso et al. (1999).

The taxonomic treatment was based on Lewis et al. (2005). The key and the descriptions were based on morphological variations found in individuals that were in the PEVRI. The illustrations were based on the collected material, and the floral parts were moisturized and drawn with a stereomicroscope. The classification of the biomes followed the Ministério do Meio Ambiente do Brazil (Brasil 2011).

\section{Results}

Overal, 23 species of Papilionoideae where recorded in the PEVRI (Table 1) distributed in 16 genera and five tribes. Phaseoleae was the most representative with 7 genera (Abrus Adans, Canavalia DC., Collaea (Loisel.) DC., Dioclea Kunth, Eriosema (DC.) Desv., Galactia P. Browne and Vigna Savi.), followed by Dalbergieae with 6 genera (Aeschynomene L., Andira Juss., Machaerium Pers., Platypodium Vogel, Stylosanthes SW., and Zornia J.F. Gmel.) and Desmodieae (Desmodium Desv.), Indigofereae (Indigofera L.) and Crotalarieae (Crotalaria L.) with one genus each.

The most representative genera were Crotalaria with four species, Aeschynomene with three species, Desmodium and Vigna with two species each, followed by Abrus, Andira, Canavalia, Collaea, Dioclea, Eriosema, Galactia, Indigofera, Machaerium, Platypodium, Stylosanthes and Zornia with one species each. The herbaceous habit occurred in six representatives, followed by subshrub and herbaceous in four, arboreous and climbing habit in three, liana in two and shrub in only one.

The predominance of herbaceous and subshrub species in open areas of the pasture and humid grasslands found in the PEVRI is equal to that of identical species widely distributed in Brazil. All the species of Papilionoideae found in the PEVRI have also been recorded in Cerrado, Seasonal Semideciduous Forest and Pantanal, except Andira inermis, which does not present a reference for Alluvial Semideciduous Forest and Collaea speciosa with no record in Pantanal.

\section{Identification key for Papilionoideae species from PEVRI}

1. Branch with leaves uni-, bi- or trifoliolate................................. 2

1 '. Branch with leaves plurifoliolate..............................................16

2. Leaves 1-2-foliolate .................................................................. 3

2'. Leaves 1-3-foliolate ............................................................4 


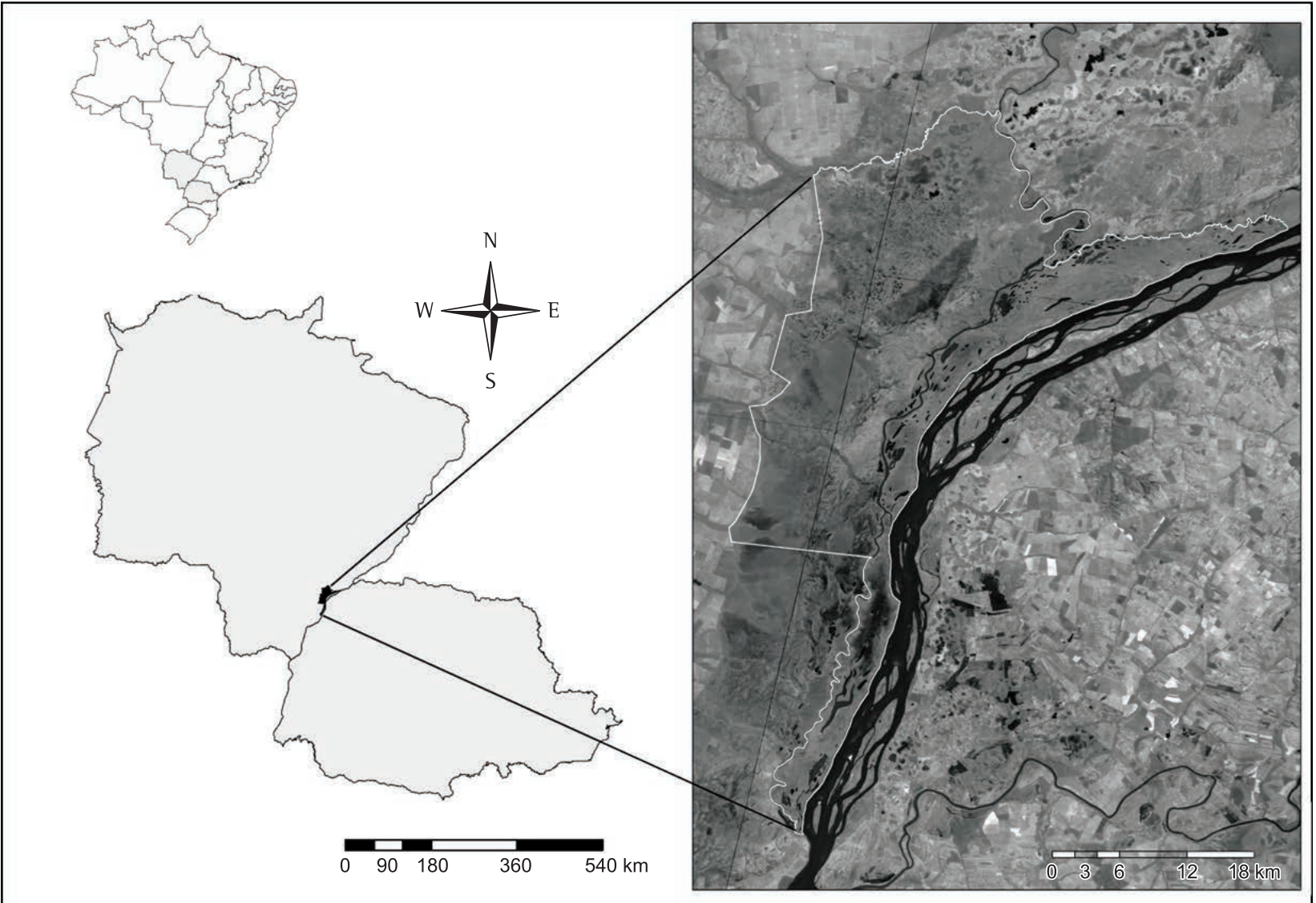

Figure 1. Location of Parque Estadual das Várzeas do Rio Ivinhema (PEVRI), Mato Grosso do Sul, Brazil.

3. Leaves 1-foliolate, the stem winged, absence of geminated bracteoles 11. Crotalaria stipularia

3'. Leaves 2-foliolate, stem without a wing, presence of two geminated bracteoles which involves each flower Zornia latifolia

4. Lianas or vines . .5

4'. Shrubs, subshrubs or herbs

...8

5. Lianas, inflorescence in panicle 14. Dioclea burkatti

5 '. Vines, inflorescence in raceme

6. Stigma lateral, with a decurrent extension .21. Vigna lasiocarpa 6'. Stigma terminal, without a decurrent extension ...7

7. Inflorescence racemose with 4-18 flora; legume 5-6 cm long, 6-9-seeded 16. Galactia striato

7'. Inflorescence racemose with 4-6 flora; legume $10.5 \mathrm{~cm}$ long, 12 -seeded 6. Canavalia mattogrossensis

8. Fruit type legume .9

8'. Fruit type loment .14

9. Legume with ovate seeds . .10

9'. Legume with reniform seeds

10. Legume up to $1.7 \mathrm{~cm}$ long, 2-seeded 15. Eriosema platycarpon 10'. Legume longer than $2 \mathrm{~cm}, 8-20$-seeded .13
11. Leaflets elliptical to lanceolate, longer than $5 \mathrm{~cm}$ .8 . Crotalaria lanceolata

11 '. Leaflets elliptical to oblong, shorter than $5 \mathrm{~cm}$ .12

12. Legume puberulent, 12 -seeded

9. Crotalaria maypurensis

12'. Legume tomentose, 28-30-seeded

10. Crotalaria micans

13. Inflorescence 2-flora calyx 5-laciniate, corolla yellow 22. Vigna longifolia

13'.Inflorescence 4-flora, calyx 4-laciniate, corolla red

7. Collaea speciosa

14. Inflorescence in capitulum, corolla yellow. 20. Stylosanthes guianensis

14'. Inflorescence racemose, corolla pink or purple

15. Villous fruit, with 2-5 articles, 2-5-seeded Desmodium barbatum

15'. Fruit hirsute to glandular, with 1-2 articles, 1-2-seeded ......12. Desmodium affine

16. Trees, samara or drupe fruit

16' Herbs, lianas, vines or subshrubs; other types of fruit

17. Leaves with fewer than 10 leaflets, leaflets lanceolate or elliptical, fruit drupe type.

5. Andira inermis

17'. Leaves with more than 15 leaflets; leaflets oblong, fruit samara type 
18. Samara with seed-chamber distal, corolla yellow 19. Platypodium elegans

18'. Samara with seed-chamber basal, corolla lilac 18. Machaerium hirtum

19. Fruit type legume .20

19'. Fruit type loment

20. Leaves 5-7-foliolate, imparipinnate; both surfaces with hirsute and malpighiaceous indumentum, inflorescence multiflora, 6-9-seeded 17. Indigofera hirsuta

20 '. Leaves 20 -foliolate, paripinnate; both surfaces with a sericeous indumentum, inflorescence 4-8 flora, 4-6-seeded ...1. Abrus praecatorius

21. Leaves 20-40-foliolate, calyx bilabiate, externally glabrous ...4. Aeschynomene sensitiva

21'. Leaves 6-10-foliolate, calyx 5-laciniate, externally hispid or sericeous..

22. Inflorescence 9-10-flora, axis $7 \mathrm{~cm}$ long, loment with $1-3$ articles 3. Aeschynomene histrix

22'. Inflorescence 2-flora, axis $0.5-1 \mathrm{~cm}$ long, loment with 5-6 articles 2. Aeschynomene falcata
1. Abrus praecatorius L. Syst. Nat. (ed. 12) 2: 472. 1767. Figure 2a

Vine; branches cylindrical; stipule and stipel linear, petiole $1-2 \mathrm{~cm}$ long, puberulent; rachis $5-10 \mathrm{~cm}$ long, sericeous. Leaves 20 -foliate, paripinnate, symmetrical leaflet, 0.7-2 × 0.4-0.9 cm, obovate; apex mucronated; base obtuse, acute; both surfaces sericeous; entire margin; venation brochidodromous, discolorous. Inflorescence pseudo-racemose, axial, 4-8 flora; peduncle $2-10 \mathrm{~cm}$ long, axis $2 \mathrm{~cm}$ long, both sericeous. Pedicel $1 \mathrm{~mm}$ long, sericeous; calyx $3 \mathrm{~mm}$ long, campanulate, 5-lacinia, $1 \mathrm{~mm}$ long, externally sericeous; corolla purple, standard 6-7 mm long, wing 5-6 mm long, keel 7-8 mm long. Stamens 10, diadelphous (9) + 1, 6-7 mm long. Gynoecium stipellate, stipe $8 \mathrm{~mm}$ long, ovary sericeous, style erect, glabrous, stigma terminal glabrous. Legume, $2.5-4.5 \times 0.5-1 \mathrm{~cm}$, oblong, apex aristate, sericeous; seeds 4-6 ovate, $4 \times 2 \mathrm{~mm}$, nut-brown.

Selected material: Brazil. Mato Grosso do Sul: Ivinhema, PEVRI, 18.IV.2009, fl. and fr., R. B. Caboco \& A. Sciamarelli 28946 (CGMS), 22 $2^{\circ}$ 56'06.3" $\mathrm{S}$ and $53^{\circ} 43^{\prime}$ 01.6” W.

Leaves with 20 leaflets, with mucronated apex, climbing habit and purple corolla allow the immediate identification of Abrus praecatorius. According to Lewis (1987), Abrus praecatorius was brought to South America, and there are records in both reef area and Atlantic Forest.

2. Aeschynomene falcata (Poir.) DC. Prodr., 2: 322. 1825. Figure 2b

Table 1. List of the sampled species of Papilionoideae in the Parque Estadual das Várzeas do Rio Ivinhema (PEVRI), Mato Grosso do Sul, Brazil, with information about the habit and the vegetation types of each species and records of occurrence in the biomes.

\begin{tabular}{|c|c|c|c|}
\hline Species & Habit & $\begin{array}{c}\text { PEVRI } \\
\text { Phyto-physionomy }\end{array}$ & Biomes \\
\hline Abrus praecatorius $\mathrm{L}$. & Liana & SDF & CAAT, CE, PA e AW ${ }^{30}$ \\
\hline Aeschynomene falcata (Poir.) Prodr. & Herb/Sub & $\mathrm{HF}$ & $\mathrm{CE}, \mathrm{PA}$ and $\mathrm{AW}{ }^{1}$ \\
\hline Aeschynomene histrix Poir. & Herb/Sub & $\mathrm{HF}$ & AMA, CAAT, CE, AW and PA ${ }^{1,2,3}$ \\
\hline Aeschynomene sensitiva $\mathrm{Sw}$. & Herb/Sub & SDF e HF & AMA, CAAT, CE, AW, PAMP e PA ${ }^{1}$ \\
\hline Andira inermis (W. Wringht) Kunth ex DC. & Tree & Alluvial SDF & AMA, CE e $\mathrm{PA}^{4}$ \\
\hline Canavalia mattogrossensis (Barb. Rodr.) Malme & Liana & SDF & $\mathrm{AMA}, \mathrm{CE}, \mathrm{AW}$ and $\mathrm{PA}^{5}$ \\
\hline Collaea speciosa (Loisel.) DC. & Sub & $\mathrm{CE}$ and $\mathrm{HF}$ & CAAT, CE and AW ${ }^{7,8,9,10}, \mathrm{AW}$ and $\mathrm{PAMP}^{6}$ \\
\hline Crotalaria lanceolata E. Mey. & Sub & FI-PA & AMA, CAAT, CE, AW, PAMP and PA ${ }^{11}$ \\
\hline Crotalaria maypurensis Kuntk & Sub & $\mathrm{HF}$ & AMA, CAAT, CE, AW, and PA ${ }^{11}$ \\
\hline Crotalaria micans Link & Sub & HF and FI-PA & AMA, CAAT, CE, AW, PAMP and PA ${ }^{1,12}$ \\
\hline Crotalaria stipularia Desv. & Herb & $\mathrm{HF}$ and FI-PA & AMA, CAAT, CE, AW and PA ${ }^{13,14}$ \\
\hline Desmodium affine Schltdl. & Herb/Sub & SDF, FI-PA and HF & AMA, CAAT, CE, AW, PAMP and PA ${ }^{15,16}$ \\
\hline Desmodium barbatum (L.) Benth. & Herb & FI-PA & AMA, CAAT, CE, AW, and $\mathrm{PA}^{5,16,17}$ \\
\hline Dioclea burkartii R.H. Maxwell & Liana & SDF & $\mathrm{CE}, \mathrm{AW}$ and $\mathrm{PA}^{18}$ \\
\hline Eriosema platycarpon Michelli & Sub & FI-PA & $\mathrm{CE}, \mathrm{AW}$ and $\mathrm{PA}^{19,20}$ \\
\hline Galactia striata (jacq.) Urb. & Liana & SDF & AMA, CAAT, CE, AW, PAMP and PA ${ }^{21}$ \\
\hline Indigofera hirsuta $\mathrm{L}$. & Herb/Sub & FI-PA & $\mathrm{CE}, \mathrm{AW}$ and $\mathrm{PA}^{21}$ \\
\hline Machaerium hirtum (Vell.) Stellfeld & Tree & FI-PA & AMA, CAAT, CE, AW and PA ${ }^{7,22,23,24}$ \\
\hline Platypodium elegans Vogel. & Tree & SDF & CE, AW, PAMP and PA ${ }^{20,25}$ \\
\hline Stylosanthes guianensis (Aubl.) Sw. & Herb/Sub & FI-PA and SF & AMA, CAAT, CE, AW and $\mathrm{PA}^{26}$ \\
\hline Vigna lasiocarpa (Mart. Ex Benth.) Verdc. & Liana & $\mathrm{HF}$ & AMA, CE, AW, PAMP and PA ${ }^{27}$ \\
\hline Vigna longifolia (Benth.) Verdc. & Herb & SDF & AMA, CAAT, CE, AW, PAMP and PA PA, $28^{27}$ \\
\hline Zornia latifolia Sm. & Herb & FI-PA & AMA, CAAT, CE, AW and $\mathrm{PA}^{7,29}$ \\
\hline
\end{tabular}

Herbaceous Herb-; Sub- Subshrub; SDF- semideciduous forest; HF- humid field; FI-PA- Field with Pastures; AMA- Amazonia; CAAT - Caatinga; CE- Cerrado; PA- Pantanal; AW - Atlantic Forest; PAMP- Pampas; SF- secondary formations. $\left({ }^{1}\right.$ Lima et al. 2006 , ${ }^{2}$ Bentham $1862,{ }^{3}$ Fernandes 1996 , ${ }^{4}$ Pennington 2003, ${ }^{5}$ Tropicos 2006, ${ }^{6}$ Ceolin \& Miotto 2009, ${ }^{7}$ Lewis 1987, ${ }^{8}$ Silvestre-Capellato \& Melhem 1997, ${ }^{9}$ Araújo et al. 2002, ${ }^{10}$ Morim 2006, ${ }^{11}$ Flores \& Miotto 2001, ${ }^{12}$ Leitão Filho et al. 1975, ${ }^{13}$ Filliettaz 2002, ${ }^{14}$ Flores 2004, ${ }^{15}$ Azevedo 1981, ${ }^{16}$ Nobre et al. 2008, ${ }^{17}$ Ducke $1949,{ }^{18}$ Maxwell 1970, ${ }^{19}$ Grear 1970, ${ }^{20}$ Dubs 1998, ${ }^{21}$ Burkart 1970, ${ }^{21}$ Moreira \& Azevedo-Tozzi 1997, ${ }^{22}$ Sartori \& Tozzi 1998, ${ }^{23}$ Bortoluzzi et al. 2004, ${ }^{24}$ Camargo 2005, ${ }^{25}$ Mendonça-Filho 1996, ${ }^{26}$ Brandão \& Costa 1979, ${ }^{27}$ Maréchal et al. 1978, ${ }^{28}$ Bentham 1859, ${ }^{29}$ Sciamarelli \& Azevedo-Tozzi 1996, ${ }^{30}$ Lewis 1987). 


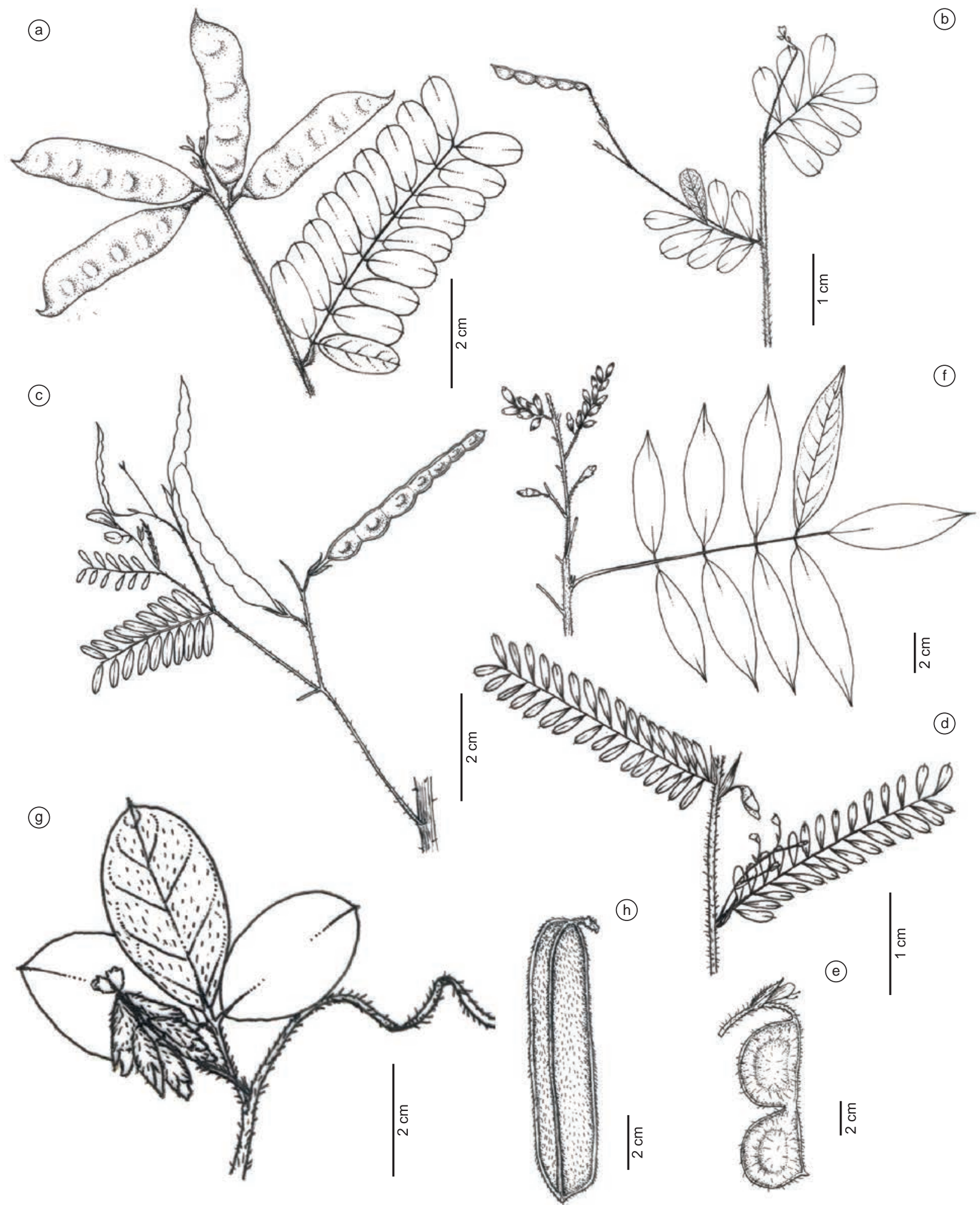

Figure 2. a. Abrus precatorius L. - part of the branch with fruit (Caboco \& Sciamarelli 01); b. Aeschynomene falcata (Poir.) Dc. - part of the branch with fruit (Pereira et al. 1592); c. Aeschynomene sensitiva Sw. - part of the branch with fruit (Pereira et al. 1628); d-e. Aeschynomene histrix Poir.; d. part of the branch with inflorescence (Pereira et al. 484); e. fruit (Pereira et al. 484); f. Andira inermis (W. Wright) Kunth ex DC. - part of the branch with inflorescence (Pereira et al. s/n); g-h. Canavalia mattogrossensis (Barb. Rodr.) Malme - g. part of the branch with inflorescence (Pereira et al. 1226); h. fruit (Pereira et al 1226).

Prostrate herb or decumbent subshrub, branches cylindrical; stipule lanceolate, striate, stipel ovate, petiole $0.2-0.3 \mathrm{~cm}$ long, rachis 0.5-1 cm long, both sericeous. Leaves 6-8-foliate, paripinnate or imparipinnate, leaflet symmetrical, 0.6-0.9 × 0.3-0.4 cm, obovate; apex mucronate; base oblique, both surfaces sericeous, entire margin, venation brochidodromous, concolorous. Inflorescence racemose, axial, 2-flora; peduncle 1-2.5 cm long, axis 0.5-1 cm long, both sericeous. Pedicel 3-5 mm long, sericeous; calyx 3-4 mm long, campanulate, 5-laciniate, 1-1.5 mm long, externally sericeous; corolla yellow, standard $4 \mathrm{~mm}$ long, wing $3.5 \mathrm{~mm}$ long, keel $3.5 \mathrm{~mm}$ long. 
Stamens 10, diadelphous (9) + 1, 2-6 mm long. Gynoecium stipellate, stipe 6-12 mm long, ovary tomentose, style curved, glabrous; stigma terminal glabrous. Loment, 3-3.3 $\times 2.5-3 \mathrm{~mm}$, ovate, 5-6 articles, obovate, apex mucronate, tomentose; seeds 5-6 ovate to reniform, $3 \times 3 \mathrm{~mm}$, nut-brown.

Selected material: Brazil. Mato Grosso do Sul: Ivinhema, PEVRI, 11.X.2006, fr. and fl., Z. V. Pereira et al. 28908 (CGMS), $22^{\circ} 55^{\prime} 14.8^{\prime \prime} \mathrm{S}$ and $53^{\circ} 39^{\prime} 13.1$ ' W.

Aeschynomene falcata is distinguished by its stipule lanceolate, leaflet obovate, stipe 6-12 mm long and loment with 5-6 tomentose articles. There are records of this species in Colombia, Bolivia, Brazil, Paraguay and the savannas, fields, rocky hillsides, high altitude areas and Chaco in Argentina (Rudd 1955). It is commonly found in cerrado and closed fields, as secondary or disturbed vegetation, along lagoon shoreline, and in flooded fields (Lima et al. 2006).

3. Aeschynomene histrix Poir., Encycl. Suppl. 4. (1): 77. 1816........ Figure 2d, e

Prostrate herb, ascending to subshrub, branches cylindrical; stipule ovate, stipel caducous, petiole $3 \mathrm{~mm}$ long, rachis $1.4 \mathrm{~cm}$ long, both hispid. Leaves 6-10-foliate, imparipinnate, leaflets symmetrical, 0.5-1 × 0.3-0.5 cm, obovate; apex mucronate, rounded; base oblique, surfaces, adaxial glabrous and abaxial hispid, entire margin, venation brochidodromous, concolorous. Inflorescence racemose, axial, 9-10 flora; peduncle $1 \mathrm{~cm}$ long, axis $7 \mathrm{~cm}$ long, hispid. Pedicel $4-6 \mathrm{~mm}$ long, hispid; calyx $2 \mathrm{~mm}$ long, campanulate, 5-laciniate, $0.5 \mathrm{~mm}$ long, externally hispid; corolla yellow, standard $2.5 \mathrm{~mm}$ long, wing $1.2 \mathrm{~mm}$ long, keel $1.5 \mathrm{~mm}$ long. Stamens 10, diadelphous (9) $+1,5.5 \mathrm{~mm}$ long. Gynoecium stipellate, stipe $1 \mathrm{~mm}$ long, ovary tomentose, style erect, hispid; stigma terminal glabrous. Loment, 3.5-4 × 2.5-3 mm, ovate, 1-3 articles, obovate, apex acute, thickly pilose; seeds 2-3, ovate to reniform, $2.5 \times 2 \mathrm{~mm}$, nut-brown.

Selected material: Brazil. Mato Grosso do Sul: Ivinhema, PEVRI, 27.III.2004, fr. and fl., Z. V. Pereira et al. 28884 (CGMS), $22^{\circ} 56^{\prime} 16.7^{\prime \prime} \mathrm{S}$ and $53^{\circ} 41^{\prime} 13.3^{\prime \prime} \mathrm{W}$.

Aeschynomene histrix is distinguished by its 6-10 foliolated leaves, obovate leaflets, apex mucronate, loment with 1, 2 or 3 articles, thick pilosity (Figure 2d). According to Lima et al. (2006), this species may be collected in areas of cerrado, mountain ridge, Elyonurus muticus grassland (caronal), Byrsonima orbignyana scrub (canjiqueiral), disturbed vegetation, secondary vegetation and lagoon shoreline.

4. Aeschynomene sensitiva Sw., Prod. 107. 1788. ........... Figure 2c

Erect herb or subshrub, branches cylindrical; stipule and stipel were not observed, petiole 3-5 cm long, hispid, rachis 30-45 mm long, glabrous. Leaves 20-40-foliate, imparipinnate, leaflets symmetrical, $0.8-1.4 \times 0.2-0.3 \mathrm{~cm}$, obovate; apex rounded, base oblique, both surfaces glabrous, entire margin, venation brochidodromous, concolorous. Inflorescence racemose, axial, 2-4 flora; peduncle 0.5-2 cm long, axis 1-1.5 cm long, both hispid. Pedicel $3 \mathrm{~mm}$ long, glabrous; calyx 5-6 mm long, bilabiate, 2-laciniate, 4-5 mm long, glabrous; corolla yellow, standard $7 \mathrm{~mm}$ long, wing $7 \mathrm{~mm}$ long, keel $8 \mathrm{~mm}$ long. Stamens 10, diadelphous (9) + 1, 5-6 mm long. Gynoecium stipellate, stipe $1 \mathrm{~mm}$ long, ovary glabrous, style erect, glabrous; stigma terminal glabrous. Loment, 6-8 ×54-5 mm, ovate, 3-7 articles, obovate, apex apiculate, seeds 3-7, ovate, $3 \times 1.5 \mathrm{~mm}$, brown.

Selected material: Brazil. Mato Grosso do Sul: Ivinhema, PEVRI, 12.X.2006, fr. and fl., Z. V. Pereira et al. 28907 (CGMS), $22^{\circ} 55^{\prime} 14.8^{\prime \prime} \mathrm{S}$ and $53^{\circ} 39^{\prime} 13.1$ ' W.

Aeschynomene sensitiva may be distinguished by its leaves with 20 to 40 obovate leaflets, rounded apex, calyx with 2 lacinia and loment with 3-7 glabrous articles. Lima et al. (2006) note that the bracts $3 \mathrm{~mm}$ long, ovate-lanceolate and flabelliform margin are important for identifying this species, but these characteristics were not observed in the material analyzed. This species occurs in flooded grasslands, swamps and disturbed vegetation (Lima et al. 2006).

5. Andira inermis (W. Wright) Kunth ex DC., Prodr. 2: 475.1825. Figure $2 \mathrm{f}$

Tree, branch cylindrical; stipule and stipel absent, petiole 4-6.5 cm long, rachis $7 \mathrm{~cm}$ long, all of them glabrous. Leaves 7-foliolate, imparipinnate, leaflets symmetrical, 3-7 × 1-2.5 cm, elliptical to lanceolate; apex acute, base diminished, surfaces glabrous, entire margin, venation brochidodromous, concolorous. Inflorescence racemose, terminal axial, multiflora; $0.5-1 \mathrm{~cm}$ long, tomentose, axis 5-15 cm long, tomentose. Pedicel 1-2 mm long, tomentose; calyx 5-6 mm long, campanulate, 5-laciniate, $1 \mathrm{~mm}$ long, tomentose; corolla yellow, standard $14 \mathrm{~mm}$ long, wing $14 \mathrm{~mm}$ long, keel 14 mm long. Stamens 10, diadelphous (9) + 1, 13-14 mm long. Gynoecium stipellate, stipe $6 \mathrm{~mm}$ long, ovary sericeous, style erect, sericeous; stigma terminal glabrous. Fruit drupe, $2.5-4.3 \times 2-4.3 \mathrm{~cm}$, ovate, glabrous (Pennington 2003).

Selected material: Brazil. Mato Grosso do Sul: Ivinhema, PEVRI, 18.IV.2009, fl., Z. V. Pereira et al. 28947 (CGMS), $22^{\circ} 55^{\prime} 14.8^{\prime \prime} \mathrm{S}$ and $53^{\circ} 39^{\prime} 13.1^{\prime \prime} \mathrm{W}$.

Andira inermis may be distinguished by its raceme inflorescence with purple flowers, standard $14 \mathrm{~mm}$ long, calyx thickly pilose and lacinia smaller than $1 \mathrm{~mm}$ in length, rounded or acute apex. There are records of this species in Seasonal Deciduous and Semideciduous Forests and cerrado (Silva 2006).

6. Canavalia mattogrossensis (Barb. Rodr.) Malme, Ark. Bot. 4(7): 9. 1905......................................................... Figure 2g, h

Vine, branches cylindrical; stipule not observed, stipel lanceolate, petiole 1-2 cm long, rachis $0.3-0.8 \mathrm{~cm}$ long, both tomentose. Leaves 3-foliate, imparipinnate, leaflet terminal asymmetrical, 3-6.5 $\times 1.6-3.5 \mathrm{~cm}$, elliptical, apex acute, retuse, base obtuse, both surfaces sericeous, tomentose, entire margin, venation brochidodromous, discolorous. Leaflet lateral 3.5-5 × 1.5-3 cm. Inflorescence racemose, axial 4-6 flora; peduncle 1-2 cm long, axis 2-3 cm long, tomentose. Pedicel 0.2-0.3 cm long, tomentose; calyx $12 \mathrm{~mm}$ long, campanulate, 5-laciniate, 2-4 mm long, externally tomentose; corolla pink, standard $30 \mathrm{~mm}$ long, wing $29 \mathrm{~mm}$ long, keel $38 \mathrm{~mm}$ long. Stamens 10, monadelphous, $35 \mathrm{~mm}$ long. Gynoecium stipellate, stipe $1 \mathrm{~mm}$ long, ovary tomentose, style erect, glabrous; stigma terminal glabrous. Legume, $10.5 \times 1.5 \mathrm{~cm}$, oblong, apex rounded, mucronate, tomentose; seeds 12 , ovate, $5 \times 8 \mathrm{~mm}$, brown spotted with black.

Selected material: Brazil. Mato Grosso do Sul: Ivinhema, PEVRI, 22.IV.2005, fr. and fl., Z. V. Pereira et al. 28900 (CGMS), $22^{\circ} 55^{\prime} 45.4 " \mathrm{~S}$ and $53^{\circ} 42^{\prime} 13.9^{\prime \prime} \mathrm{W}$.

Canavalia mattogrossensis is readily distinguished by its keel petals which are bigger ( $38 \mathrm{~mm}$ long) than, or equal to, the standard length (30 mm long) and racemose inflorescences with nodules along its axis. According to Tropicos (2006), there are records in Seasonal Semideciduous Forest and cerrado.

7. Collaea speciosa (Loisel.) DC., Mem. Leg. 6: 245. 1825. ........... Figure 3a, b

Subshrub, branches cylindrical; stipule and stipel absent, petiole $0.2 \mathrm{~cm}$ long, tomentose, rachis absent. Leaves 3 -foliate, imparipinnate, leaflets symmetrical, $3-5.5 \times 0.6-0.8 \mathrm{~cm}$, lanceolate, apex acute, diminished base, entire margin, venation eucamptodromous, surfaces, adaxial glabrous and abaxial tomentose, discolorous. Inflorescence racemose, axial, 4-flora, peduncle $0.2-0.3 \mathrm{~cm}$ long, axis $0.1-0.2 \mathrm{~cm}$ long, tomentose. Pedicel $1-1.5 \mathrm{~cm}$ long, tomentose; calyx $16 \mathrm{~mm}$ 
Papilionoideae do PEVRI

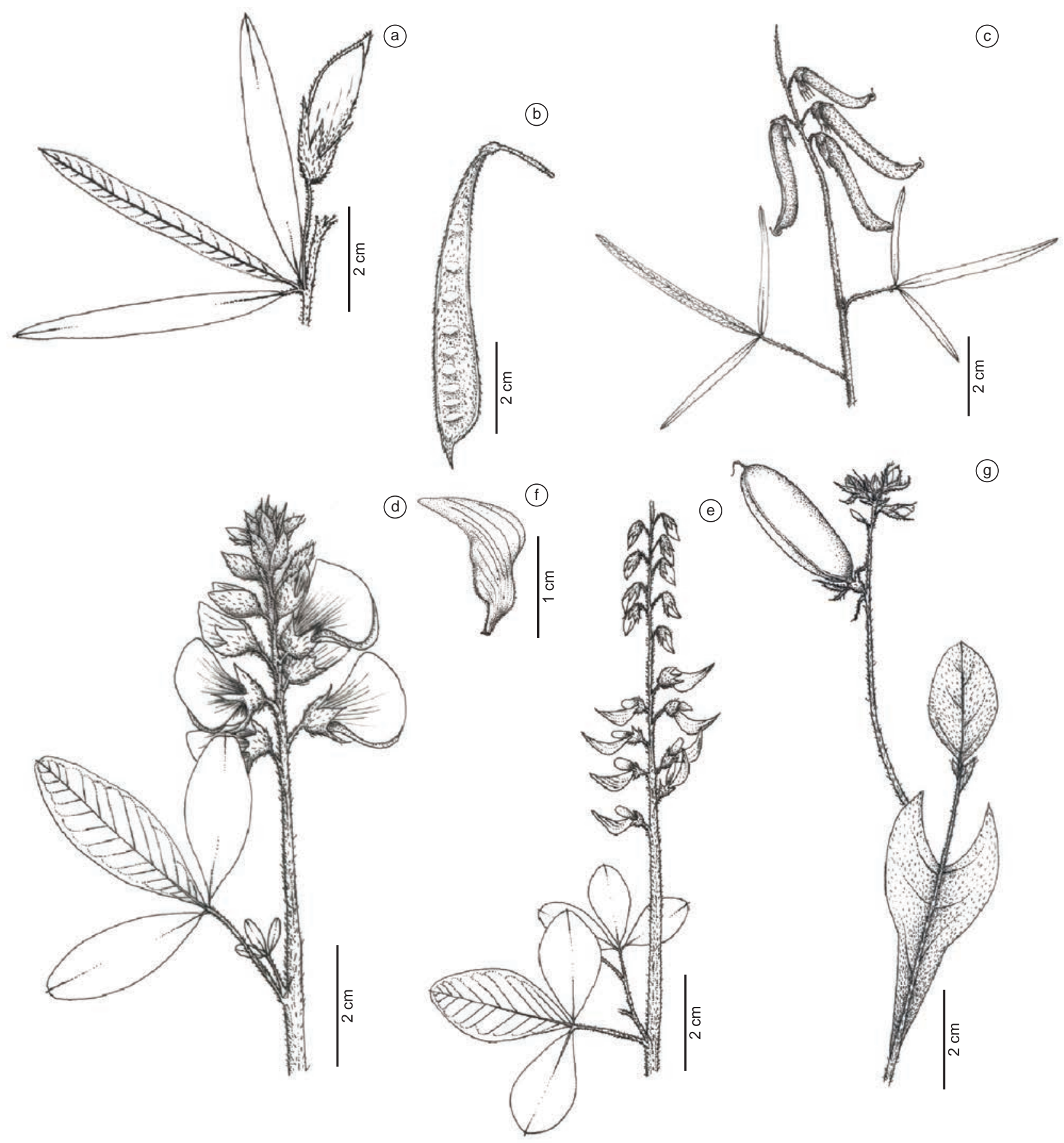

Figure 3. a-b. Collaea speciosa (Loisel.) DC. a. - part of the branch with flower; b. fruit (Pereira et al. 1197); c. Crotalaria lanceolata E. Mey.- part of the branch with inflorescence (Caboco \& Sciamarelli 37); d. Crotalaria maypurensis Kunth- part of the branch with inflorescence (Pereira et al. 344); e-f. Crotalaria micans Link - branch with inflorescence; f. standard. (Caboco \& Sciamarelli 23); g. Crotalaria stipularia Desv. - branch with inflorescence and fruit (Caboco \& Sciamarelli 27).

long, campanulate, 4-laciniate, 8-10 mm long, tomentose; corolla red, standard $33 \mathrm{~mm}$ long, wing $30 \mathrm{~mm}$ long, keel $25 \mathrm{~mm}$ long. Stamens 10, diadelphous (9) +1, 23-25 mm long. Gynoecium stipellate, stipe $1 \mathrm{~mm}$ long, ovary tomentose, style erect, glabrous; stigma terminal glabrous. Legume, $6.5 \times 0.7 \mathrm{~cm}$, oblong, apex apiculate, tomentose; seeds 10-20, ovate, $2 \times 1 \mathrm{~mm}$, nut-brown, brown.

Selected material: Brazil. Mato Grosso do Sul: Ivinhema, PEVRI, 21.IV.2005, fr. and fl., Z. V. Pereira et al. 28904 (CGMS), $22^{\circ} 55^{\prime} 14.8^{\prime \prime} \mathrm{S}$ and $53^{\circ} 39^{\prime} 13.1^{\prime \prime} \mathrm{W}$.
Collaea speciosa may be noted as a subshrub by its 3-foliate leaves, lanceolate leaflets, inflorescence with short peduncle (0.3-0.2 cm long), red flowers and calyx tomentose. According to Ceolin \& Miotto (2009), this species is generally isolated, occupying wooded fields, gallery forest and roadsides.

8. Crotalaria lanceolata E. Mey., Comm. Pl. Afr. Austr. 1: 24. 1836

Figure $3 \mathrm{c}$

Subshrub, branches cylindrical; stipule and stipel not observed, petiole $1-3 \mathrm{~cm}$ long, puberulent, rachis $0.1 \mathrm{~cm}$ long, puberulent. Leaves 
3-foliate, leaflet terminal asymmetrical, 1.5-6.5 × 0.4-0.5 cm long, elliptical to lanceolate, apex acute, apiculate, base acute, surfaces, adaxial glabrous and abaxial puberulent, entire margin, venation camptodromous, discolorous, leaflet lateral 5-14 $\times 0.4-0.5 \mathrm{~cm}$ long. Inflorescence racemose, terminal, multiflora; peduncle $3-6 \mathrm{~cm}$ long, puberulent, axis 6-10 cm long, puberulent. Pedicel $2 \mathrm{~cm}$ long, puberulent; calyx 3-4 mm long, campanulate, 5-laciniate, $1 \mathrm{~mm}$ long, externally puberulent; corolla yellow, standard $9 \mathrm{~mm}$ long, wing $10 \mathrm{~mm}$ long, with brown grooves, keel $11 \mathrm{~mm}$ long. Stamens 10, monadelphous, 8-9 mm long. Gynoecium stipellate, stipe $1 \mathrm{~mm}$ long, ovary puberulent, style curved, sericeous, stigma terminal glabrous. Legume, 3-3.5 $\times 0.4-0.5 \mathrm{~cm}$ long, oblong, apex apiculate, puberulent; seeds 16-20, reniform, $2 \times 2 \mathrm{~mm}$, orange or fawn.

Selected material: Brazil. Mato Grosso do Sul: Ivinhema, PEVRI, 18.IV.2009, fr. and fl., R. B. Caboco \& A. Sciamarelli 28926 (CGMS), 22 51'05.8' S and 53 ${ }^{\circ} 39^{\prime} 18.2^{\prime \prime} \mathrm{W}$.

Crotalaria lanceolata is distinguished by its leaflets lanceolate, abaxial puberulence, inflorescence axis $6-10 \mathrm{~mm}$ long and wing with brown grooves.

9. Crotalaria maypurensis Kunth, Nov. Gen. Sp. 6: 403. 1824. ...... Figure 3d

Subshrub, branches cylindrical; stipule linear, elliptical, stipel not observed, petiole 2-4 cm long, rachis $0.1-0.2 \mathrm{~cm}$ long, both puberulent. Leaf 3 -foliate, leaflet terminal asymmetrical, $3.5-4 \times 1.2-1.8 \mathrm{~cm}$, elliptical to oblong, apex acute and apiculate, diminished base, surfaces, adaxial glabrous and abaxial puberulent, entire margin, venation camptodromous, discolorous, leaflet lateral $2-3.5 \times 0.8-1.2 \mathrm{~cm}$ long. Inflorescence racemose, terminal, multiflora, peduncle $6-8 \mathrm{~cm}$ long, axis 10-32 cm long, puberulent. Pedicel $1.0 \mathrm{~cm}$ long, puberulent; calyx 8-10 mm long, campanulate, 5-laciniate, 5-7 mm long, sericeous; corolla yellow, standard 17-20 mm long, wing $18 \mathrm{~mm}$ long, with fawn grooves, keel $18 \mathrm{~mm}$ long. Stamens 10 , monadelphous, $16 \mathrm{~mm}$ long. Gynoecium stipellate, stipe $1 \mathrm{~mm}$ long, ovary sericeous; style curved, glabrous; stigma terminal glabrous. Legume, $3.2 \times 1 \mathrm{~cm}$, oblanceolate, apex apiculate, puberulent; seeds 12 , reniform, $3 \times 3 \mathrm{~mm}$, nut-brown.

Selected material: Brazil. Mato Grosso do Sul: Ivinhema, PEVRI, 23.XI.2004, fr. and fl., Z. V. Pereira et al. 28876 (CGMS),

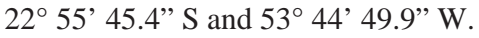

Crotalaria maypurensis may present a variation in the shape of its leaflets, i.e., from elliptical to oblong, inflorescence axis 10-32 cm long, standard 17-20 mm long, with fawn grooves. In Brazil, it is frequently found in cerrado areas (Flores \& Miotto 2001).

10. Crotalaria micans Link, Enum. Hort. Berol. Alt. 2: 228, 1822. Figure $3 \mathrm{e}, \mathrm{f}$

Subshrub, branches cylindrical; stipule elliptical, stipel absent, petiole $2-3 \mathrm{~cm}$ long, rachis $0.3 \mathrm{~cm}$ long, tomentose. Leaves 3 -foliate, leaflets symmetrical, $2.5-5 \times 1.6-2.8 \mathrm{~cm}$ long, oblong to elliptical, apex apiculate, cuneated base, surfaces, adaxial glabrous and abaxial sericeous, entire margin, venation camptodromous, discolorous. Inflorescence racemose, terminal, multiflora, peduncle $6-8 \mathrm{~cm}$ long, axis $15-35 \mathrm{~cm}$ long, both pubescent. Pedicel $0.4-0.5 \mathrm{~cm}$ long, pubescent; calyx 6-8 mm long, campanulate, 5-laciniate, 3-4 mm long, externally sericeous; corolla yellow, standard 12-13 mm long, wing 11-14 mm long, without grooves, keel 18-21 mm long. Stamens 10, monadelphous, 12-13 mm long. Gynoecium stipellate, stipe $1 \mathrm{~mm}$ long, ovary sericeous, style curved, sericeous, stigma terminal ciliate. Legume, $2.5-3.5 \times 0.4-0.5 \mathrm{~cm}$, linear to oblong, apex apiculate, tomentose in the external region of the placentation; glabrous in the opposite position; seeds 28-30, reniform, $3 \times 2 \mathrm{~mm}$, fawn.
Selected material: Brazil. Mato Grosso do Sul: Ivinhema, PEVRI, 18.IV.2009, fr. and fl., R. B. Caboco \& A. Sciamarelli 28927 (CGMS), 22 $2^{\circ} 55^{\prime} 45.4^{\prime \prime} \mathrm{S}$ and 53 $3^{\circ} 44^{\prime} 49.9^{\prime \prime} \mathrm{W}$.

The inflorescence axis of Crotalaria micans is longer $(15-35 \mathrm{~cm})$ than that of $C$. maypurensis, and it presents smaller flowers (12-13 mm long).

\section{Crotalaria stipularia Desv., J. Bot. Agric. 3: 76. 1814. Figure3g}

Erect herb, winged stem, branches cylindrical; stipule and stipel not observed, internode wing 2-6 cm long. Leaf 1-foliate, leaflet symmetrical, $2.5-4.5 \times 1.5-3 \mathrm{~cm}$ long, elliptical to obovate, apex retuse to apiculate, diminished base, both surfaces hispid, entire margin, venation camptodromous, discolorous. Inflorescence racemose, axial, peduncle $4-6 \mathrm{~cm}$ long, rachis $5-12 \mathrm{~cm}$ long, both tomentose. Pedicel 0.1-0.4 cm long, tomentose; calyx 7-10 mm long, campanulate, 5-laciniate, lacinium 5-7 mm long, sericeous; corolla yellow, standard 5-6 mm long, wing $5 \mathrm{~mm}$ long, without grooves, keel $7 \mathrm{~mm}$ long. Stamens 10, monadelphous, 7-8 mm long. Gynoecium stipellate, stipe $1 \mathrm{~mm}$ long, ovary sericeous, style curved, hispid near the stigma, stigma terminal ciliate. Legume, $2-3.5 \times 0.8-1 \mathrm{~cm}$, oblong, apex apiculate, glabrous; seeds 21-27, reniform, $3 \times 2 \mathrm{~mm}$, nut-brown.

Selected material: Brazil. Mato Grosso do Sul: Ivinhema, PEVRI, 18.IV.2009, fr. and fl., R. B. Caboco \& A. Sciamarelli 28880 (CGMS), 22 $2^{\circ}$ ' $50.2^{\prime \prime} \mathrm{S}$ and $53^{\circ} 38^{\prime} 40.7^{\prime}$ 'W.

Crotalaria stipularia may be distinguished by its winged stem, unifoliate leaves, elliptical leaflet, obovate, with hispid trichomes on both surfaces. In South America, this species is found from Venezuela and Guiana to Argentina (Filliettaz 2002, Flores \& Miotto 2001). It is found in "restinga", riparian forest, cerrado, open fields, "campo rupestre" and sandy, swampy and disturbed places (Filliettaz 2002, Flores 2004).

\section{Desmodium affine Schltdl., Linnaea 12: 312. 1838. Figure 4a, b}

Prostrate herb or subshrub, branches cylindrical; stipule ovatelanceolate, stipel lanceolate, petiole $0.6-1.8 \mathrm{~cm}$ long, sericeous, rachis 0.2-0.5 cm long, sericeous. Leaves 3-foliate, leaflets symmetrical 3-6.2 $\times 1-2 \mathrm{~cm}$, elliptical, apex acute, base obtuse, cuneate, entire margin, venation brochidodromous, both surfaces sericeous, discolorous. Inflorescence racemose, terminal and axial, multiflora; peduncle $3-5 \mathrm{~cm}$ long, axis $6-10 \mathrm{~cm}$ long, both hirsute to sericeous. Pedicel 2-5 mm long, hirsute; calyx $3 \mathrm{~mm}$ long, campanulate, 5-laciniate, $2 \mathrm{~mm}$ long, hirsute; corolla pink, standard $6 \mathrm{~mm}$ long, wing $5 \mathrm{~mm}$ long, keel $5 \mathrm{~mm}$ long. Stamens 10, diadelphous, (9) + 1, $4 \mathrm{~mm}$ long. Gynoecium stipellate, stipe $1 \mathrm{~mm}$ long, ovary hirsute, style erect, glabrous; stigma terminal glabrous. Loment, $6 \times 4 \mathrm{~mm}$, oblong, 1-2 articles, obovate, apex apiculate, hirsute to glandular; seeds $1-2$, ovate to reniform, $1.5 \times 2 \mathrm{~mm}$, white.

Selected material: Brazil. Mato Grosso do Sul: Ivinhema, PEVRI, 12.IX.2006, fr. and fl., Z. V. Pereira et al. 28910 (CGMS),

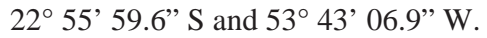

Desmodium affine is distinguished by its ovate-lanceolate stipule, elliptical leaflets, loment with 1-2 articles, obovate, with hirsuteglandular trichomes. It is found in woods, cerrado, grasslands, "campo rupestre", but it is mainly found in humid and shadowy areas (Azevedo 1981, Nobre et al. 2008).

13. Desmodium barbatum (L.) Benth., Pl. Jungh. 2: 224. 1852. ..... Figure 4c, d

Prostrate herb, branches cylindrical, stipule and stipel lanceolate, petiole $5-13 \mathrm{~mm}$ long, rachis $1-3 \mathrm{~cm}$ long, both sericeous. Leaf 1-3-foliate, leaflet asymmetrical 1-2.6 $\times 0.5-1.8 \mathrm{~cm}$, elliptical to obovate; apex obtuse to rounded, base rounded, cuneate, adaxial surface glabrous, villous, abaxial villous to sericeous, entire margin, venation brochidodromous, concolorous, lateral leaflet 
Papilionoideae do PEVRI

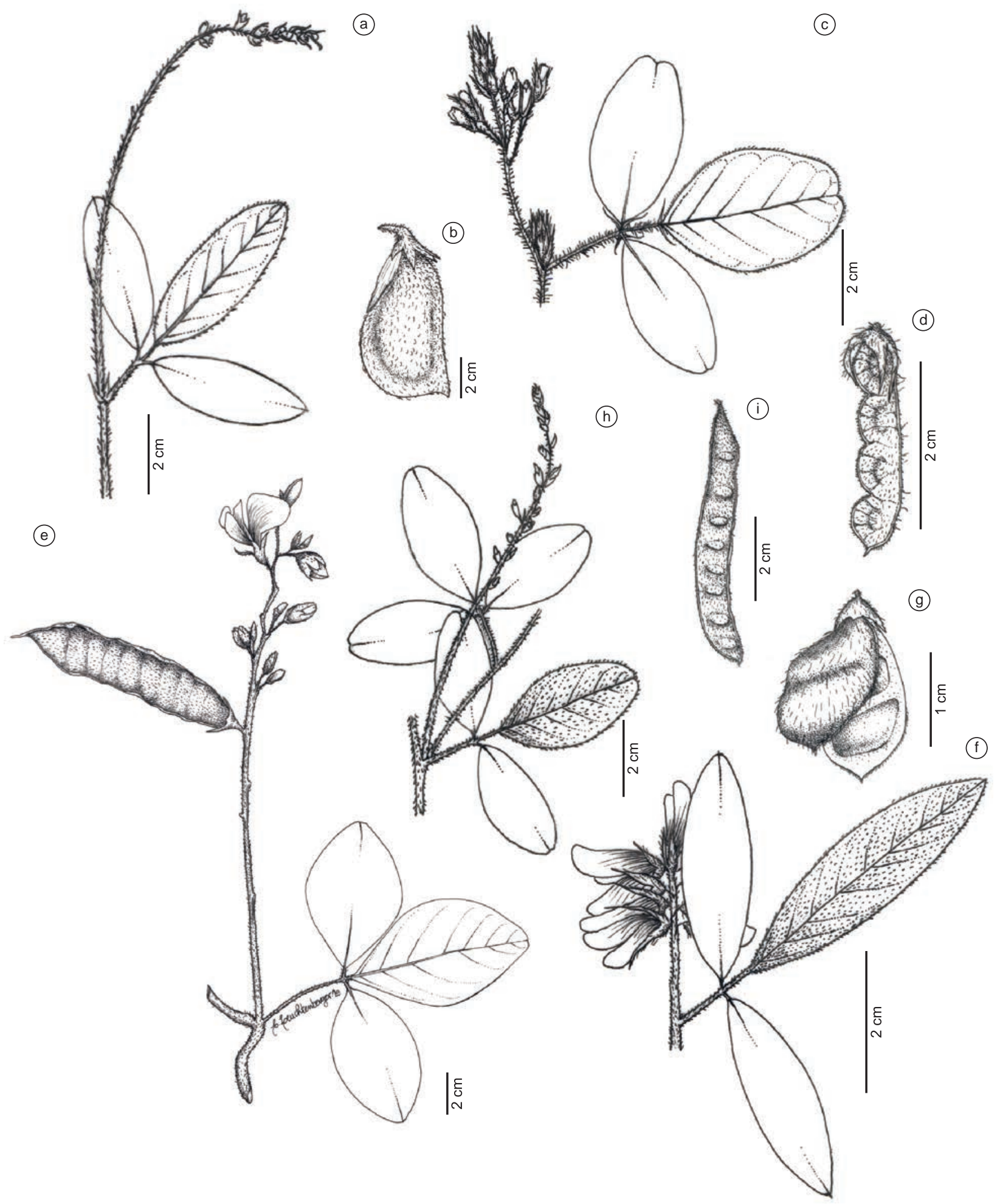

Figure 4. a-b. Desmodium affine Schltdl. - a. part of the branch with inflorescence; b. fruit. (Pereira et al. 1643); c-d. Desmodium barbatum (L.) Benth. - c. part of the branch with inflorescence (Pereira et al. 444); d. fruit. (Pereira et al. 444); e. Dioclea burkartii R.H. Maxwell - part of the branch with inflorescence and fruit. (Pereira et al. 277); f-g. Eriosema platycarpon Micheli - f. part of the branch with inflorescence; g. fruit. (Caboco \& Sciamarelli 34); h-i. Galactia striata (Jacq.) Urb. h- part of the branch with inflorescence; i. fruit. (Pereira et al. 1235).

$0.9-1.9 \times 0.6-1 \mathrm{~cm}$. Inflorescence racemose, terminal to axial, multiflora; peduncle 1-2 cm long, villous, axis 1-3 cm long, villous. Pedicel 6-8 mm long, sericeous; calyx $4 \mathrm{~mm}$ long, campanulate, 5-laciniate, $3 \mathrm{~mm}$ long, villous; corolla purple, standard $6 \mathrm{~mm}$ long, wing $5 \mathrm{~mm}$ long, keel $5 \mathrm{~mm}$ long. Stamens 10, diadelphous (9) + 1, $5 \mathrm{~mm}$ long. Gynoecium stipellate, stipe $1 \mathrm{~mm}$ long, ovary tomentose, style erect, glabrous; stigma glabrous. Loment, 4-5 × 3-4 mm, oblong, 2-5 articles, ovate, apex apiculate, villous; seeds 2-5, ovate, $2 \times 1 \mathrm{~mm}$, black.

Selected material: Brazil. Mato Grosso do Sul: Ivinhema, PEVRI, 27.III.2004, fr. and fl., Z. V. Pereira et al. 28944 (CGMS), $22^{\circ} 56^{\prime} 06.3^{\prime \prime} \mathrm{S}$ and $53^{\circ} 43^{\prime} 01.6^{\prime \prime} \mathrm{W}$. 
Desmodium barbatum may be identified by its uni- to trifoliate leaf, elliptical and obovate leaflets present on the same plant, villous calyx and loment, which may vary from 2-5 articles. This species may be found in cerrado, swampy grasslands, dirty grasslands and degraded area (Ducke 1949, Tropicos 2006, Nobre et al. 2008).

14. Dioclea burkartii R.H. Maxwell, Darwiniana 16(1-2): 413. 1970. Figure $4 \mathrm{e}$

Liana branches cylindrical; stipule ovate, stipel lanceolate, petiole $2-3 \mathrm{~cm}$ long, rachis $0.5 \mathrm{~cm}$ long, both sericeous. Leaves 3-foliate, leaflet terminal asymmetrical 6-7.5 × 4-4.5 cm long, ovate to elliptical; apex acute, base oblique, both surfaces sericeous, entire margin, venation brochidodromous, discolorous, lateral leaflet 4.5-6.5 × 3-4.5 cm. Inflorescence racemose nodose, axial, 15-26-flora; peduncle $4.5 \mathrm{~cm}$ long, axis of the inflorescence $20-25 \mathrm{~cm}$, both sericeous. Pedicel 5-7 mm long, sericeous; calyx $14 \mathrm{~mm}$ long, campanulate, 5-laciniate, $5.5 \mathrm{~mm}$ long, sericeous; corolla lilac, standard $25 \mathrm{~mm}$ long, sericeous, wing $23 \mathrm{~mm}$ long, keel 20-23 mm long. Stamens 10, monadelphous or pseudomonadelphous, $33 \mathrm{~mm}$ long. Gynoecium stipellate, stipe $1 \mathrm{~mm}$ long, ovary tomentose, style curved, glabrous; stigma puberulent. Legume, 8.5-10 × 1.5-2.2 cm, oblong, apex apiculate, tomentose; seeds 9, ovate, $13 \times 7 \mathrm{~mm}$, nut-brown.

Selected material: Brazil. Mato Grosso do Sul: Ivinhema, PEVRI, 06.IX.2003, fr. and fl., Z. V. Pereira et al. 28875 (CGMS), $22^{\circ} 55^{\prime} 45.4 " \mathrm{~S}$ and $53^{\circ} 44^{\prime} 49.9^{\prime \prime} \mathrm{W}$.

The main characteristics of Dioclea burkartii are the ovate and elliptical leaflets, acute apex, inflorescence racemose with nodes evident, lilac corolla and tomentose fruit 8 to $10 \mathrm{~cm}$ long. Database records of this species are few in number. It is only noted in Mato Grosso do Sul and Paraná (Maxwell 1970).

15. Eriosema platycarpon Micheli, Mem. Soc. Phys. Genev. 28(7): 34. 1883. Figure 4f, $g$

Subshrub, branches cylindrical; stipule and stipel lanceolate, petiole $0.5 \mathrm{~mm}$ long, rachis $1.5 \mathrm{~cm}$ long, both tomentose. Leaves 3-foliate, leaflets symmetrical $0.8-3.4 \times 1.5-2.5 \mathrm{~cm}$ long, elliptical; apex acute, base oblique, tomentose, entire margin, venation brochidodromous, discolorous. Inflorescence racemose, axial, 8-11-flora; peduncle $2.5 \mathrm{~cm}$, tomentose, axis $2.5-4 \mathrm{~cm}$ long, tomentose. Pedicel $6 \mathrm{~mm}$ long, tomentose; calyx $5 \mathrm{~mm}$ long, campanulate, 5-laciniate, $3 \mathrm{~mm}$ long, tomentose; corolla yellow, standard $15 \mathrm{~mm}$ long, wing $10 \mathrm{~mm}$ long, keel $15 \mathrm{~mm}$ long. Stamens 10, diadelphous $(9)+1,15 \mathrm{~mm}$ long. Gynoecium stipellate, stipe $1 \mathrm{~mm}$ long, ovary tomentose, style erect, tomentose; stigma terminal, tomentose. Legume, 1.5-1.7 × 1-1.2 cm, oblong, apex mucronate, tomentose; seeds 2, ovate, 5-4 × 3-4 mm, greenish brown.

Selected material: Brazil. Mato Grosso do Sul: Ivinhema, PEVRI, 19.IV.2009, fr. and fl., R. B. Caboco \& A. Sciamarelli 28891 (CGMS), $22^{\circ} 5546.3$ " S and 53० 43' 07.4" W.

Eriosema platycarpon is distinguished by its elliptical leaflets, tomentose, discolorous and relatively small fruit $(1.5-1.7 \times 1-1.2 \mathrm{~cm})$. It is found along the cerrado edges and in the transition of woody cerrado and riparian forest and parks, as well as sandy and silty soils (Cristaldo, unpublished data).

16. Galactia striata (Jacq.) Urb., Symb. Antill. . 2(2): 320. 1900. .. Figure 4h, i

Vine, branches cylindrical; stipule and stipel lanceolate, petiole 0.6-3 cm long, rachis 2-5 $\mathrm{mm}$ long, both sericeous. Leaves 3 -foliate, leaflet terminal symmetrical 2.5-6 × 1-2.4 cm, elliptical; apex apiculate, base acute, both sericeous, entire margin, venation brochidodromous, discolorous, leaflet lateral 2.2-4.5 $\times 0.9-2 \mathrm{~cm}$. Inflorescence racemose, axial, 4-18-flora; peduncle 1-4 cm long, axis
$6.5 \mathrm{~cm}$ long, both sericeous. Pedicel 1-2 mm long, sericeous; calyx $5 \mathrm{~mm}$ long, campanulate, 5-laciniate, 2-3 mm long, sericeous; corolla purple, standard $11.5 \mathrm{~mm}$ long, wing 9-10 mm long, keel $10 \mathrm{~mm}$ long. Stamens 10, diadelphous (9) + 1, 10-11 mm long. Gynoecium stipellate, stipe $1 \mathrm{~mm}$ long, ovary tomentose, style erect, glabrous; stigma terminal, glabrous. Legume, 5-6 × 0.6-0.7 cm, oblong, apex apiculate, sericeous; seeds 6-9, ovate, $4 \times 4 \mathrm{~mm}$, black.

Selected material: Brazil. Mato Grosso do Sul: Ivinhema, PEVRI, 22.IV.2005, fr. and fl., Z. V. Pereira et al. 28897 (CGMS), $22^{\circ} 55^{\prime} 14.8^{\prime \prime} \mathrm{S}$ and $53^{\circ} 39^{\prime} 13.1$ ' W.

Galactia striata is mainly characterized by its lanceolate stipule and stipel, venation brochidodromous, discolorous, and legume 5-6 $\times$ 0.6-0.7 cm, oblong. According to Burkart (1970), there are records of this species in the Americas, from the southeast of the United States to Argentina.

17. Indigofera hirsuta L., Sp. Pl. 2: 751. 1753. .............. Figure 5a

Herb or subshrub, branches cylindrical; stipule lanceolate, stipel not observed, petiole 1-2 cm long, rachis $2.5-3.0 \mathrm{~cm}$ long, both hirsute. Leaves 5-7-foliate, imparipinnate, leaflet terminal asymmetrical, 1-2.5 $\times 0.6-1.5 \mathrm{~cm}$, obovate; apex mucronate, diminished base, both hirsute and malpighiaceous, entire margin, venation not evident, leaflet lateral 0.9-1.4 × 0.4-0.8 cm. Inflorescence racemose, axial, multiflora; peduncle $1 \mathrm{~mm}$ long, axis $4-5.5 \mathrm{~cm}$ long; calyx $4 \mathrm{~mm}$ long, campanulate, 5-laciniate, $1 \mathrm{~mm}$ long, tomentose; corolla pink, standard 3-4 mm long, wing $4 \mathrm{~mm}$ long, keel $5 \mathrm{~mm}$ long. Stamens10, diadelphous (9) + 1, 4-4.2 mm long. Gynoecium stipellate, stipe $1 \mathrm{~mm}$ long, ovary glabrous, style erect, glabrous; stigma terminal glabrous. Legume, $0.2-1.6 \times 0.2-1.2 \mathrm{~cm}$, cylindrical, tomentose, apex acute; seeds $6-9$, ovate, $1 \times 1 \mathrm{~mm}$, black and nut-brown.

Selected material: Brazil. Mato Grosso do Sul: Ivinhema, PEVRI, 27.III.2004, fr. and fl., Z. V. Pereira et al. 28879 (CGMS), $22^{\circ} 52^{\prime} 50.2^{\prime \prime} \mathrm{S}$ and $53^{\circ} 38^{\prime} 40.7^{\prime \prime} \mathrm{W}$.

The peculiarities of Indigofera hirsuta include the hirsute and malpighiaceous indumentum on the branches, leaflets, peduncles and floral axis, leaves with 5 to 7 leaflets, lanceolate stipules and cylindrical fruit. According to Moreira \& Azevedo-Tozzi (1997), it is commonly found in disturbed areas, secondary vegetation and sandy soils.

18. Machaerium hirtum (Vell.) Stellfeld, Tribuna Farm., Bras. 12i. 132.1944 Figure $5 b$

Tree, branches cylindrical; stipule lanceolate, rectilinear, spinescent, stipel not observed, petiole $0.5-1 \mathrm{~cm}$ long, rachis 8-12 cm long, both tomentose. Leaves 40-foliate, imparipinnate, leaflets symmetrical, $1-1.3 \times 0.3-0.4 \mathrm{~cm}$, oblong; apex mucronate, diminished base, both surfaces glabrous, entire margin, venation craspedodromous, discolorous. Inflorescence paniculate, terminal to axial, multiflora; axis of inflorescence $11-16 \mathrm{~cm}$ long, villous (Polido \& Sartori 2007). Pedicel 8.8-10.6 mm long, villous; calyx $2.6 \mathrm{~mm}$ long, campanulate, 5-laciniate; corolla lilac; standard 6.9-9.3 ×5.9-7.6 mm, wing 7.6-9 × 3.3-4.7 mm long, keel 7.1-9.6 × 4.5-5.1 mm. Stamens 10 , monadelphous. Gynoecium not stipellate, ovary velutinous, style erect, stigma glabrous. Samara cultriform, 4.5-5.4 × 0.9-1.2 cm, apex obtuse, apiculate, glabrescent; seed-chamber basal, brownish, tomentose, $2-3.4 \times 1-1.2 \mathrm{~cm}$.

Selected material: Brazil. Mato Grosso do Sul: Ivinhema PEVRI, 19.IV.2009, fr., R. B. Caboco \& A. Sciamarelli 28931 (CGMS), $22^{\circ} 56^{\prime} 15.3^{\prime \prime} \mathrm{S}$ and $53^{\circ} 41^{\prime} 01.9^{\prime \prime} \mathrm{W}$.

Macherium hirtum is distinguished by its rectilinear, spinescent stipules, oblong leaflets and arboreous habit. It is found in several phyto-physyonomy of Cerrado, in sandy and clayish soils, in Seasonal Semideciduous and Deciduous Forests and dense Ombrophylous Forest (Polido \& Sartori 2007). 


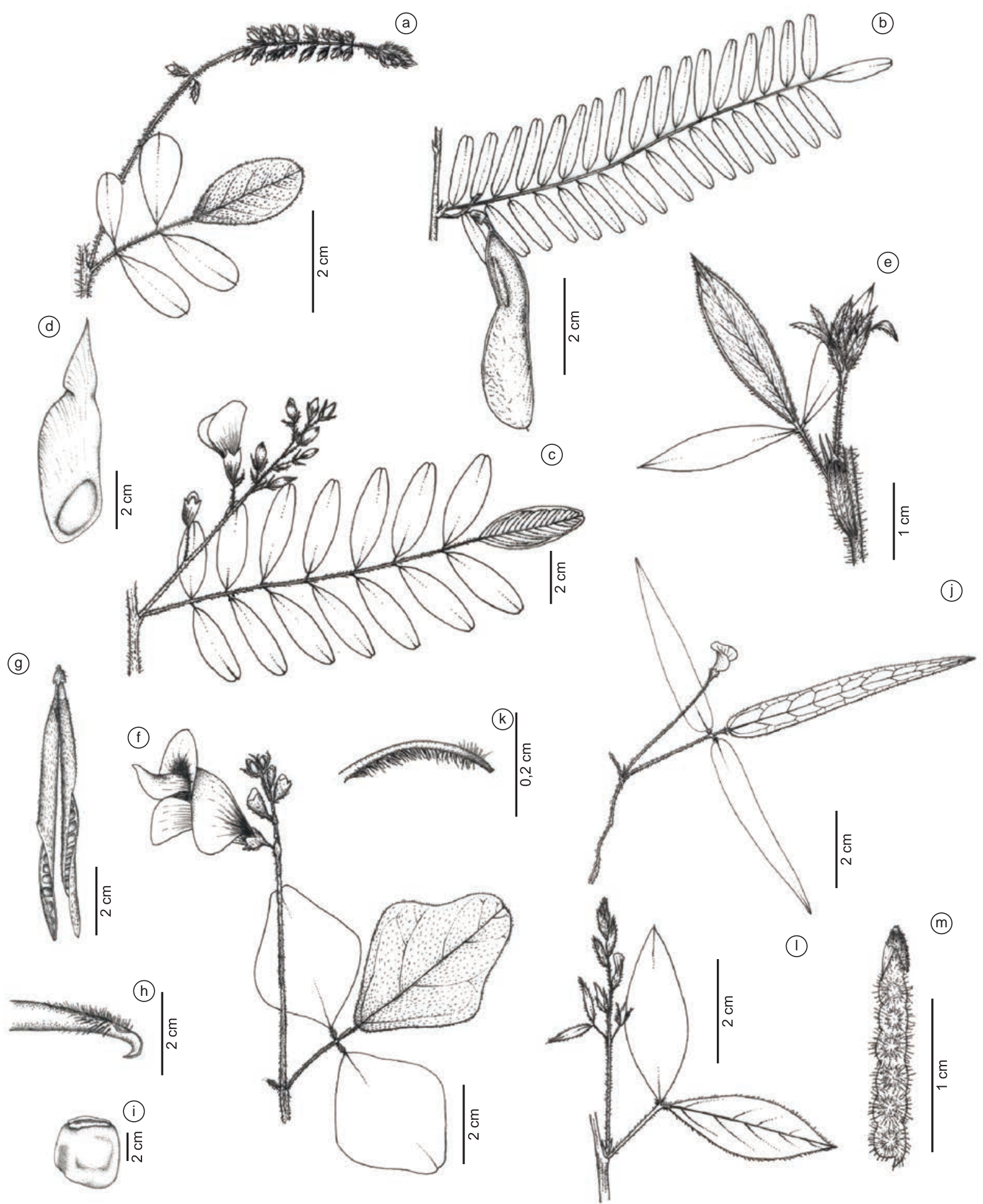

Figure 5. a. Indigofera hirsuta L. - part of the branch with inflorescence (Pereira et al. 443); b. Machaerium hirtum (Vell.) Stellfeld - leaf and fruit; c-d. Platypodium elegans Vogel. - c. part of the branch with inflorescence; d. Fruit (Caboco \& Sciamarelli 1731); e. Stylosanthes guianensis (Aubl.) Sw. - branch with inflorescence (Caboco \& Sciamarelli 39); f-i. Vigna lasiocarpa (Mart. former Benth.) Verdc. - f. Branch with inflorescence; g. fruit; h. style; i. seed (Caboco \& Sciamarelli 1731); j-k. Vigna longifolia (Benth.) Verdc. - j. branch with flower; k. style (Pereira et al. 1627); 1-m. Zornia latifolia Sm. - 1. branch with inflorescence; $\mathrm{m}$. fruit (Pereira et al. 1456).

19. Platypodium elegans Vogel., Linnaea 11: 420. 1837.

Figure 5c, d

Tree, branches cylindrical, stipule and stipel caducous, petiole 1-16 cm long, sericeous, rachis $8.5-12 \mathrm{~cm}$ long, sericeous. Leaves 17 -foliate, pinnate, leaflets symmetrical, $4.3 \times 1-1.5 \mathrm{~cm}$, elliptical to oblong; apex obcordate, diminished base, both surfaces sericeous, entire margin, venation eucamptodromous, discolorous. Inflorescence racemose, axial, 20-flora, peduncle $1.8-2.2 \mathrm{~cm}$ long, axis $8.5-9 \mathrm{~cm}$ long, both sericeous. Zygomorphic flower; pedicel $6 \mathrm{~mm}$ long, sericeous; calyx $9.5 \mathrm{~mm}$ long, campanulate, 5-lacinia; lacinium $2 \mathrm{~mm}$ long, sericeous; corolla yellow; standard $20 \mathrm{~mm}$, wing $19 \mathrm{~mm}$ long, keel $14 \mathrm{~mm}$ long. Androecium heterodynamous, stamens 10, 
diadelphous (9) + 1, 10-12 mm long. Gynoecium stipellate, stipe $6 \mathrm{~mm}$ long, ovary sericeous, style curved, glabrous, stigma terminal puberulent. Samara elliptical, $6.4 \times 2.3 \mathrm{~cm}$, apex acute, glabrous; seed-chamber distal, fawn, glabrate, $2 \times 1 \mathrm{~cm}$.

Selected material: Brazil. Mato Grosso do Sul: Ivinhema, PEVRI, 11.XI.2004, fl. and fr., R. B. Caboco \& A. Sciamarelli 28912, idem, 19/IV/2009, fr., 28942 (CGMS), 22 55' 59.6” $\mathrm{S}$ and $53^{\circ} 40^{\prime} 23.5^{\prime \prime} \mathrm{W}$.

Platypodium elegans presents 17 -foliolate, pinnate leaves, obcordate apex, both surfaces sericeous, and samara $6.4 \times 2.3 \mathrm{~cm}$. This species has been reported to occupy areas of Seasonal Semideciduous and Deciduous Forests and cerrado (Mendonça-Filho 1996, Dubs 1998).

20. Stylosanthes guianensis (Aubl.) Sw., Kongl. Vetensk. Acad. Nya Handl. 10: 301. 1789. Figure 5e

Herb or subshrub, branches cylindrical; stipule lanceolate, stipel not observed, petiole $0.3-1 \mathrm{~cm}$ long, villous, rachis $1-2 \mathrm{~mm}$ long, tomentose. Leaf 3-foliate, leaflets symmetrical, 0.4-2.9 $\times 0.3-1.9 \mathrm{~cm}$, lanceolate; apex acute, diminished base, both surfaces sericeous, entire margin, venation eucamptodromous, discolorous. Inflorescence in capitulum, fasciculate, terminal or axial, peduncle $3.5-5 \mathrm{~cm}$ long, villous, axis $0.5-1 \mathrm{~cm}$ long, villous. Flower without pedicel; calyx $7 \mathrm{~mm}$ long, villous, 5-laciniate, 1-3 mm long; corolla yellow, standard $5 \mathrm{~mm}$ long, wing $4 \mathrm{~mm}$ long, keel $4 \mathrm{~mm}$ long. Stamens 10, monadelphous, $8 \mathrm{~mm}$ long. Gynoecium stipellate, stipe $1 \mathrm{~mm}$ long, ovary puberulent, with terminal appendix, style erect, glabrous; stigma terminal, glabrous. Loment, 1 article, $4 \times 2 \mathrm{~mm}$, rounded, puberulent; seed 1, ovate, $2.4 \times 1 \mathrm{~mm}$, fawn.

Selected material: Brazil. Mato Grosso do Sul: Ivinhema, PEVRI 23.III.2004, fl., Z. V. Pereira et al. 28877 (CGMS); idem 19/IV/2009, fr., R. B. Caboco \& A. Sciamarelli 28921 (CGMS), 22 $2^{\circ} 55^{\prime} 46.3^{\prime \prime} \mathrm{S}$ and $53^{\circ} 43^{\prime} 07.4^{\prime \prime} \mathrm{W}$.

Stylosanthes guianensis may be identified by its striped stipules, eucamptodromous venation, discolorous leaflets, inflorescence in capitulum and fasciculate, absence of pedicel and lomentum with one article. According to Costas et al. (2008), this species may be found in cerrado, and dirty grasslands.

21. Vigna lasiocarpa (Mart. ex Benth.)Verdc., Kew Bull. 24(3): 539. 1970. Figure $5 f, i$

Vine, branches cylindrical; stipule elliptical to lanceolate, stipel linear, petiole $1.5-3 \mathrm{~cm}$ long, sericeous, glabrous, rachis $0.5-1 \mathrm{~cm}$ long, sericeous. Leaves 3 -foliate, leaflet terminal asymmetrical 2.5-3.5 $\times 1-2.5 \mathrm{~cm}$, trullate to elliptical; apex acute, stalked base, oblique, adaxial surface sericeous, abaxial surface glabrous, entire margin, venation brochidodromous, discolorous, leaflet lateral 2-3.5 $\times 1-2.2 \mathrm{~cm}$. Inflorescence racemose, axial, multiflora; peduncle 3-6.5 cm long, sericeous. Pedicel 1-2 mm long, glabrous; calyx $5 \mathrm{~mm}$ long, campanulate, 5-laciniate, 1-2 mm long, sericeous; corolla purple, standard 13-22 mm long, wing 15-29 mm long, keel $20 \mathrm{~mm}$ long. Stamens 10, diadelphous (9) $+1,25 \mathrm{~mm}$ long. Gynoecium stipellate, stipe $1 \mathrm{~mm}$ long, ovary sericeous, style curved with trichomes near the terminal stigma, glabrous. Legume, $7 \times 0.5 \mathrm{~cm}$, oblong, apex acute, sericeous; seeds $8-10$, oblong, $4 \times 3 \mathrm{~mm}$, brown marbled with black and beige.

Selected material: Brazil. Mato Grosso do Sul: Ivinhema, PEVRI, 19.IV.2009, fl. and fr., R. B. Caboco \& A. Sciamarelli 28940 (CGMS), 22 55' 50.6" S and 53 42'19.4" W.

Vigna lasiocarpa may be identified by its habit, elliptical stipule to lanceolate, multiflora inflorescence, oblong legume, and marbled seeds, both black and beige. It is associated with humid environments and flooded areas (Maréchal et al. 1978).
According to Snak et al. (2011) this species is recognized by its auriculated stipule with different lobes and sericeous indument. It is commonly confused with $\mathrm{V}$. longifolia which is distinguished by its elongated style and a stigmatic region.

22. Vigna longifolia (Benth.) Verdc., Kew Bull. 24:541 (1970)....... Figure 5j, k

Prostrate herb, branches cylindrical; stipule expanded below point of insertion, basis auriculate, stipule and stipel lanceolate, petiole $1.5-3.5 \mathrm{~cm}$ long, strigose to tomentose, rachis $0.2-0.3 \mathrm{~mm}$ long, strigose. Leaf 3-foliate, leaflets symmetrical 2-6.5 $\times 0.3-0.6 \mathrm{~cm}$, lanceolate; apex acute, base obtuse, both surfaces strigose, entire margin, venation brochidodromous, concolorous. Inflorescence racemose, axial, 2-flora; peduncle $4 \mathrm{~cm}$ long, axis $0.3 \mathrm{~cm}$ long, both strigose; calyx $3 \mathrm{~mm}$ long, campanulate, 5-laciniate, $1 \mathrm{~mm}$ long, sericeous; corolla yellow, orange, standard $16 \mathrm{~mm}$ long, wing $10 \mathrm{~mm}$ long, keel 17 mm long. Stamens 10, diadelphous (9+1), 14-15 mm long. Gynoecium stipellate, stipe $1 \mathrm{~mm}$ long, ovary tomentose, style erect, sericeous near the lobated lateral stigma. Legume, 6-6.6 $\times$ 0.4-0.7 cm, linear, apex acute, sericeous; seeds 12, ovate, $4 \times 2 \mathrm{~mm}$, nut-brown with white aril.

Selected material: Brazil. Mato Grosso do Sul: Ivinhema, PEVRI, 12.IX.2006, fl. and fr., Z. V. Pereira et al. 28909 (CGMS), $22^{\circ} 51^{\prime} 11.1^{\prime \prime} \mathrm{S}$ and $53^{\circ} 39^{\prime} 18.2^{\prime \prime} \mathrm{W}$.

Vigna longifolia is distinguished by its stipules with auriculate basis and equal lobes, corolla yellow to orange, petals from the keel twisted and lateral stigma. It is frequently confused with V. lasiocarpa, which differs by its non-elongated style and its stigmatic region (Snak et al. 2011).

23. Zornia latifolia Sm., Cycl.39: 4. 1819....................Figure 51, m

Prostrate herb, branches cylindrical; stipule and stipel not observed, petiole 1-2 cm long, sericeous. Leaves 2-foliate, bipinnate, leaflets symmetrical $3.3-3.5 \times 1-1.3 \mathrm{~cm}$, elliptical to lanceolate; apex acute, base oblique, diminished, both surfaces sericeous, entire margin, venation brochidodromous, concolorous. Spike inflorescence, axial, 6-12-flora; peduncle 1-2 cm long, sericeous, rachis $2-6 \mathrm{~cm}$ long, sericeous. Pedicel absent; bracteoles geminated, calyx $4 \mathrm{~mm}$ long, bilabiate, 2-laciniate, 2-3 mm long, sericeous; corolla yellow, standard $6 \mathrm{~mm}$ long, wing $3 \mathrm{~mm}$ long, keel $4 \mathrm{~mm}$ long. Stamens 10 , monadelphous, 5-6 mm long. Gynoecium stipellate, stipe $1 \mathrm{~mm}$ long, ovary tomentose, style erect, tomentose, lateral stigma, glabrous. Loment, 6-7 articles, $3 \times 2 \mathrm{~cm}$, ovate, uncinate; seeds 6-7, ovate and reniform, $1.5 \times 1.5 \mathrm{~mm}$, yellow.

Selected material: Brazil. Mato Grosso do Sul: Ivinhema, PEVRI, 16.VII.2005, fl. and fr., Z. V. Pereira et al. 28898 (CGMS), $22^{\circ} 56^{\prime} 06.3^{\prime \prime} \mathrm{S}$ and $53^{\circ} 43^{\prime} 01.6^{\prime \prime} \mathrm{W}$.

Zornia latifolia may be distinguished by its bifoliate leaves and loment with 6-7 uncinate articles, presence of two geminated bracteoles which involves each flower in the inflorescence.

\section{Discussion}

About $35 \%$ of species studied in PEVRI are distributed in Seasonal Semideciduous Forest and 26\% occur only in this type of forest, which suggests the importance of preserving them for the maintenance of species richness such as the Papilonoideae. Andira inermis is the only species recorded in PEVRI whose habit is Alluvial Semideciduous Forest. Dioclea burkatii recorded only for the Pantanal has expanded its distribution since it occurs in the Seasonal Semideciduous Forest of the park. Among the 23 recorded species of Papilonoideae in PEVRI, nine of them are found in humid grasslands, and the most representatives are Aeschynomene, Crotalaria, Desmodium and Vigna. The occurrence of Aeschynomene falcata, 
Aeschynomene histrix, Crotalaria maypurensis and Vigna lasiocarpa denote the capacity of these species to survive in flooded soil, since they were not observed in other environments in PEVRI.

When the studies of Aeschynomene (Lima et al. 2006), Desmodium (Nobre et al. 2008), Machaerium (Polido \& Sartori 2007, 2011) and Stylosanthes (Costas et al. 2008) are compared, these authors mentioned Aeschynomene falcata, A. histrix, A. sensitiva, Desmodium affine, D. barbatum, Machaerium hirtum and Stylosanthes guianensis, respectively, as common to those sampled in PEVRI, which occupy cerrado, flooded grasslands and disturbed areas. For Sciamarelli (unpublished data), who studied areas similar to those from PEVRI, no common species were present.

PEVRI is the second largest unit of conservation in Mato Grosso do Sul and its flora is influenced by its formations which constitutes the basins from Paraná and Paraguai rivers. Ours results are an important tool for understanding the diversity of flora in the conservation area in southern Mato Grosso do Sul, which is occupied by the westernmost border of the Atlantic biome.

\section{Acknowledgements}

We thank the UFMS Master's Program in Plant Biology for conceding the structure for the realization of the work. We also thank CAPES for providing a scholarship to the first author and CNPq for a scholarship of Scientific Initiation (PIBIC) to the second author.

\section{References}

ARAÚJO, G.M., BARBOSA, A.A.A., AMARAL, A.F. \& ARANTES, A.A. 2002. Composição florística de veredas no Município de Uberlândia, MG. Rev. Bras. Bot. 25:475-493.

AZEVEDO, A.M.G. 1981. O gênero Desmodium Desv. no Brasil: Considerações taxonômicas. Dissertação de mestrado, Universidade Estadual de Campinas, Campinas, 315p.

BARROSO, G.M., MORIM, M.P., PEIXOTO, A.L. \& ICHASO, C.L.F. 1999. Frutos e sementes: morfologia aplicada a sistemática de dicotiledôneas. Universidade Federal de Viçosa, Viçosa, 443p.

BENTHAM, G. 1859. Leguminosae. In Flora Brasiliensis (C.F. Von Martius, S. Endlicher \& I. Urban, eds.). CRIA, v. 15 find. Fleischer, Monachii, Lipsiae.

BENTHAM, G. 1862. Papilionaceae. In Flora Brasiliensis (C.F. Von Martius, eds.). CRIA, v.15, n.1, p.291-9. Monachii.

BORTOLUZZI, R.L.C., CARVALHO-OKANO, R.M., GARCIA, F.C.P. \& TOZZI, A.M.G.A. 2004. Leguminosae, Papilionoideae no Parque Estadual do Rio Doce, Minas Gerais, Brasil. II: árvores e arbustosescandentes. Acta Bot. Bras. 18(1):49-71.

BRANDÃO, M. \& COSTA, N.M.S. 1979. O gênero Stylosanthes Sw. no Brasil. Epamig, Belo Horizonte.

BRASIL. Ministério Do Meio Ambiente - MMA. 2011. Geoprocessamento/ Biomas i3GEO. http://mapas.mma.gov.br/i3geo/ (último acesso em 08/03/2011).

BURKART, A. 1970. Las Leguminosas-Faseolas argentinas de los géneros Mucuna, Dioclea, y Camptosema. Darwiniana 16(1-2):175-218.

CAMARGO, R.A. 2005. A tribo Dalbergieae (Leguminosae-Faboideae) no estado de Santa Catarina, Brasil. Dissertação de mestrado, Universidade Federal do Rio Grande do Sul, Porto Alegre, 131p.

CAMPOS, J.B. \& SOUZA, M.C. 1997. Vegetação. In A planície de inundação do alto rio Paraná: aspectos físicos, biológicos e socioeconômicos(A.E.A.M. Vazzoler, A.A. Agostinho \& N.S. Hahn, eds.). Editora da Universidade Estadual de Maringá, Maringá.

CEOLIN, G.B. \& MIOTTO, S.T.S. 2009. O gênero Collaea DC. (Leguminosae, Papilionoideae) na Região Sul do Brasil. Acta Bot. Bras. 23(4):991-998.

COSTAS, L.C., SARTORI, A.L. \& POTT, A. 2008. Estudo taxonômico de Stylosanthes (Leguminosae- Papilionoideae- Dalbergieae) em Mato Grosso do Sul, Brasil. Rodriguésia 59(3):547-572.
DUBS, B. 1998. Leguminosae - Papilionoideae. In Prodromus florae matogrossensis (B. Dubs, ed.). Betrona Verlag, Kusnacht, p.140-148.

DUCKE, A. 1949. As leguminosas da Amazônia brasileira: notas sobre a flora neotrópica - II. Instituto Agronômico do Norte, Belém, n.18, p.200-202.

FERNANDES, A. 1996. O táxon Aeschynomene no Brasil. EUFC, Fortaleza.

FILLIETTAZ, A.M. 2002. Estudos taxonômicos de espécies de Crotalaria sect. Calycinae Wight \& Arn. (Leguminosae-Papilionoideae-Crotalarieae) no Brasil. Dissertação de mestrado, Universidade Estadual de Campinas, Campinas.

FLORES, A.S. 2004. Taxonomia, números cromossômicos e química das espécies de Crotalaria L. (Leguminosae, Papilionoideae) no Brasil. Tese de doutorado, Universidade Estadual de Campinas, Campinas.

FLORES, A.S. \& MIOTTO, S.T.S. 2001. O gênero Crotalaria L. (Leguminosae - Faboideae) na Região Sul do Brasil. Iheringia 55:189247.

FORZZA, R.C., BAUMGRATZ, J.F., BICUDO, C.E.M., CARVALHO JÚNIOR, A.A., COSTA, A., COSTA, D.P., HOPKINS, M., LEITMAN, P.M., LOHMANN, L.G., MAIA, L.C., MARTINELLI, G., MENEZES, M., MORIM, M.P., COELHO, M.A.N., PEIXOTO, A.L., PIRANI, J.R., PRADO, J., QUEIROZ, L.P., SOUZA, V.C., STEHMANN, J.R., SYLVESTRE, L.S., WALTER, B.M.T. \& ZAPPI, D., eds. 2010. Catálogo de plantas e fungos do Brasil. Andrea Jakobsson Estúdio, Jardim Botânico do Rio de Janeiro, Rio de Janeiro, v.1, 871p.

FRANCO, A.A. \& FARIA, S.M. 1997.The contribution of $\mathrm{N}_{2}$-fixing tree legumes to land reclamation and sustainability in the tropics. Soil Biol. Biochem. 29(5-6):897-903. http://dx.doi.org/10.1016/S00380717(96)00229-5

GREAR, J.J.W. 1970. A revision of the American species of Eriosema (Leguminosae-Lotoideae). Mem. New York Botanical Garden 20(3):1-98.

GUEDES-BRUNI, R.R., MORIM, M.P., LIMA, H.C. \& SYLVESTRE, L.S. 2002. Inventário Florístico. In Manual Metodológico para Estudos Botânicos na Mata Atlântica (L.S. Sylvestre \& M.M.T. Rosa, orgs.). Seropédica, Rio de Janeiro, p.24-49.

HARRIS, J.G. \& HARRIS, M.W. 1994. Plant identification terminology: an ilustred glossary. Spring Lake Publishing, Utah, 198p.

HERRERA, M.A., SALAMANCA, C.P. \& BAREA, J.M. 1993. Inoculation of woody legumes with selected arbuscular mycorrhizal fungi and rhizobia to recover desertified mediterranean ecosystems. Appl. Environ. Microbiol. 59(1):129-133.

KÖPPEN, W. 1948. Climatologia: con un estudio de los climas de la tierra. Fondo de Cultura Econômica, México, p.1-479.

LEITÃO FILHO, H.F., ARANHA, C. \& BACCHI, O. 1975. Plantas invasoras de culturas no Estado de São Paulo. Hucitec, São Paulo, v.2.

LEWIS, G.P. 1987. Legumes of Bahia. Royal Botanic Gardens, Kew, 369p.

LEWIS, G.P., SCHRIRE, B., MACKINDER, B. \& LOCK, M. 2005. Legumes of the World. Royal Botanic Gardens, Kew.

LIMA, L.C.P., SARTORI, A.L.B. \& POTT, V.J. 2006. Aeschynomene L. (Leguminosae, Papilionoideae, Aeschynomeneae) no Estado de Mato Grosso do Sul. Hoehnea 33 (4):419-453.

LIMA, H.C., QUEIROZ, L.P., MORIM, M.P., SOUZA, V.C., DUTRA, V.F., BORTOLUZZI, R.L.C., IGANCI, J.R.V., FORTUNATO, R., VAZ, A.M.S., SOUZA, E.R., FILARDI, F.L., GARCIA, F.C.P., FERNANDES, J.M., MARTINS-DA-SILVA, R.C.V., FORTUNA-PEREZ, A.P., MANSANO, V.F., MIOTTO, S.T.S., TOZZI, A.M.G.A., MEIRELES, J.E., LIMA, L.C.P., FLORES, A.S., TORKE, B.M., PINTO, R.B., LEWIS, G.P., BARROS, M.J.F., RIBEIRO, R.D., RODRIGUES, R.S., PENNINGTON, T., KLITGAARD, B., SCALON, V.C., CARDOSO, D.B.O.S., COSTA, L.C., SILVA, M.J., MOURA, T.M., BARROS, L.A.V.; SILVA, M.C.R., QUEIROZ, R.T., SARTORI, Â.L.B. \& CAMARGO, R.A. 2010. Fabaceae. In Catálogo de plantas e fungos do Brasil (R.C. Forzza, J.F. Baumgratz, C.E.M. Bicudo, A.A. Carvalho Júnior, A. Costa, D.P. Costa, M. Hopkins, P.M. Leitman, L.G. Lohmann, L.C. Maia, G Martinelli, M. Menezes, M.P. Morim, M.A.N. Coelho, A.L. Peixoto, J.R. Pirani, J. Prado, L.P. Queiroz, V.C. Souza, J.R. Stehmann, L.S. Sylvestre, B.M.T. Walter \& D. Zappi, eds.). Andrea Jakobsson Estúdio, Jardim Botânico do Rio de Janeiro, Rio de Janeiro, v.2, p.989-1102. 
Caboco, R.B. et al.

MARÉCHAL, R., MASCHERPA, J.M. \& STAINER, F. 1978. Étude taxonomique d'un groupe complex d'espéces des genres Phaseolus et Vigna (Papilionaceae) sur la base de données morphologiques et polliniques, traitées par l'analyse informatique. Boissiera 28:1-273.

MATO GROSSO DO SUL (Estado). Secretaria de Estado de Meio Ambiente - SEMA. 2001. Decreto 9.278, de 17 de dezembro de 1998. Decreto de criação do Parque das Várzeas do Rio Ivinhema. Oficial de Mato Grosso do Sul (DOEMS), Campo Grande, dez. 2006.

MAXWELL, R.H. 1970. A new species of Dioclea (Leguminosae) from Argentina. Darwiniana 16(1-2):413-416.

MENDONÇA-FILHO, C.V. 1996. Braúna, angico, jacarandá e outras leguminosas de Mata Attântica: Estação Biológica de Caratinga, Minas Gerais. Fundação Botânica Margaret Mee; Fundação Biodoiversitas, Belo Horizonte, 100p.

MOREIRA, J.L.A. \& AZEVEDO-TOZZI, A.M.G. 1997. Indigofera L. (Leguminosae, Papilionoideae) no Estado de São Paulo, Brasil. Rev. Bras. Bot. 20(1):97-117.

MORIM, M.P. 2006. Leguminosae arbustivas e arbóreas da Floresta Atlântica do Parque Nacional do Itatiaia, sudeste do Brasil: padrões de distribuição. Rodriguésia 57:27-45.

NOBRE, A.V.M., SARTORI, A.L.B. \& RESENDE, U.M. 2008. As espécies de Desmodium Desv. (Leguminosae-Papilionoideae-Desmodieae) ocorrentes no Mato Grosso do Sul. Iheringia, Sér. Bot. 63(1):37-67.

NOGUCHI, D.K., PETINE, G.N. \& SARTORI, A.L.B.S. 2009. Florística e síndromes de dispersão de espécies arbóreas em remanescentes de Chaco de Porto Murtinho, Mato Grosso do Sul. Rodriguesia 60(2):353-365.

OLIVEIRA, M.J.R. \& LUZARDO, R. 2000. Geologia Estrutural. In Programa Levantamentos Geológicos Básicos do Brasil: Caracaraí. (Companhia de Pesquisa de Recursos Minerais - CPRM, ed.). CPRM, Brasília. Folhas NA.20-Z-B e NA.20-Z-D (integrais), NA.20-Z-A, NA.21-Y-A, NA.20Z-C e NA.21-Y-C (parciais). Escala 1:500.000. E.

PENNINGTON, R.T. 2003. Monograph of Andira (LeguminosaePapilionoideae). Syst. Bot. Monogr. 64:1-143. http://dx.doi. org/10.2307/25027903
POLIDO, C.A. \& SARTORI, A.L.B. 2007. O genêro Machaerium (Leguminosae-Papilionoideae-Dalbergieae) no Pantanal Brasileiro. Rodriguésia 58(2):313-329.

POLIDO, C.A. \& SARTORI, A.L.B. 2011. Machaerium (Leguminosae, Papilionoideae, Dalbergieae) nos estados de Mato Grosso e Mato Grosso do Sul, Brasil. Rodriguésia 62(1).

POTT, A. \& POTT, V.J. 1994. Plantas do Pantanal. Embrapa, Brasília, 320p.

QUEIROZ, L.P. 2009. Leguminosas da Caatinga. Universidade Estadual de Feira de Santana, 467p.

RADFORD, A.E., DICKISON, W.C., MASSEY, J.R. \& BELL, C.R. 1974. Vascular plant systematics. Harper \& Row, New York, 891p.

RUDD, V.E. 1955. The American species of Aeschynomene. Bul. U.S. Nat. Herb. 32:1-172.

SARTORI, A.L.B. \& TOZZI, A.M.G.A. 1998. As espécies de Machaerium Pers. (Leguminosae - Papilionoideae - Dalbergieae) ocorrentes no estado de São Paulo. Rev. Bras. Bot. 21(3):211-246.

SCIAMARELLI, A. \& AZEVEDO-TOZZI, A.M.G. 1996. Zornia J. F. GMEL. (Leguminosae - Papilionoideae - Aeschynomeneae) no estado de São Paulo. Acta Bot. Bras. 10(2).

SILVA, R.R. 2006. Leguminosae no Planalto Residual do Urucum, oeste do Pantanal de Mato Grosso do Sul, Brasil: inventário, taxonomia e similaridade florística. Tese de doutorado, Universidade Estadual de Campinas, Campinas, 318p.

SILVESTRE-CAPELLATO, M. \& MELHEM, T.S. 1997. Flora polínica da Reserva do Parque Estadual das Fontes do Ipiranga (São Paulo, Brasil). Família 81 - Leguminosae. Hoehnea 24:119-167.

SNAK, C., MIOTTO, S.T.S. \& GOLDENBERG, R. 2011. Phaseolinae (Leguminosae, Papilionoideae, Phaseoleae) no estado do Paraná, Brasil. Rodriguésia 62(3):695-716.

TROPICOS. http://www. tropicos.org (último acesso em 01/01/2006).

VELOSO, H.P., RANGEL FILHO, A.L.R. \& LIMA, J.C.A. 1991.A classificação da vegetação brasileira adaptada a um sistema universal. IBGE, Rio de Janeiro. 